\title{
In vitro and in vivo efficacy of the novel oral proteasome inhibitor NNU546 in multiple myeloma
}

\author{
Hui Zhou ${ }^{1,}$, Meng Lei ${ }^{2,}{ }^{*}$, Wang Wang 3 , Mengjie Guo ${ }^{3}$, Jia Wang ${ }^{4}$, Haoyang Zhang ${ }^{1}$, Li Qiao ${ }^{3}$, \\ Huayun Feng ${ }^{2}$, Zhaogang Liu ${ }^{4}$, Lijuan Chen ${ }^{5}$, Jianhao Hou ${ }^{3}$, Xueyuan Wang ${ }^{1}$, Chenxi Gu ${ }^{6}$, Bo \\ Zhao ${ }^{6}$, Evgeny Izumchenko7,*, Ye Yang ${ }^{3,8,}{ }^{*}$, Yongqiang Zhu ${ }^{1,4, *}$ \\ ${ }^{1}$ College of Life Science, Nanjing Normal University, Nanjing 210046, PR China \\ ${ }^{2}$ College of Science, Nanjing Forestry University, Nanjing 210037, PR China \\ ${ }^{3}$ School of Medicine and Holistic Integrative Medicine, Nanjing University of Chinese Medicine, Nanjing 210023, \\ PR China \\ ${ }^{4}$ Jiangsu Chia Tai Fenghai Pharmaceutical Co. Ltd., Nanjing 210046, PR China \\ ${ }^{5}$ The 1st Affiliated Hospital of Nanjing Medical University, Nanjing 210029, PR China \\ ${ }^{6}$ School of Chemistry and Materials Science, Nanjing Normal University, Nanjing 210046, PR China \\ ${ }^{7}$ Department of Medicine, Section of Hematology and Oncology, University of Chicago, Chicago, IL 60637, USA \\ ${ }^{8}$ The 3rd Affiliated Hospital, Nanjing University of Chinese Medicine, Nanjing 210023, PR China \\ *Equal contribution
}

Correspondence to: Yongqiang Zhu, Evgeny Izumchenko, Ye Yang; email: zhyqscu@hotmail.com, https://orcid.org/00000003-0137-5907; izumchen@medicine.bsd.uchicago.edu, 290422@njucm.edu.cn

Keywords: proteasome inhibitor, multiple myeloma, oral drug, mechanism research

Received: June 17, $2020 \quad$ Accepted: August 15, $2020 \quad$ Published: November 16, 2020

Copyright: (C) 2020 Zhou et al. This is an open access article distributed under the terms of the Creative Commons Attribution License (CC BY 3.0), which permits unrestricted use, distribution, and reproduction in any medium, provided the original author and source are credited.

\section{ABSTRACT}

Proteasome inhibition demonstrates highly effective impact on multiple myeloma (MM) treatment. Here, we aimed to examine anti-tumor efficiency and underlying mechanisms of a novel well tolerated orally applicable proteasome inhibitor NNU546 and its hydrolyzed pharmacologically active form NNU219. NNU219 showed more selective inhibition to proteasome catalytic subunits and less off-target effect than bortezomib ex vivo. Moreover, intravenous and oral administration of either NNU219 or NNU546 led to more sustained pharmacodynamic inhibitions of proteasome activities compared with bortezomib. Importantly, NNU219 exhibited potential anti-MM activity in both MM cell lines and primary samples in vitro. The anti-MM activity of NNU219 was associated with induction of G2/M-phase arrest and apoptosis via activation of the caspase cascade and endoplasmic reticulum stress response. Significant growth-inhibitory effects of NNU219 and NNU546 were observed in 3 different human MM xenograft mouse models. Furthermore, such observation was even found in the presence of a bone marrow microenvironment. Taken together, these findings provided the basis for clinical trial of NNU546 to determine its potential as a candidate for MM treatment.

\section{INTRODUCTION}

The generation and degradation of intracellular proteins is essential for maintaining cellular function [1]. Protein degradation mediated via the ubiquitin-proteasome pathway (UPP) is an important mechanism regulating cellular protein homeostasis [2, 3]. UPP degrades proteins via specific attachment of ubiquitin. Proteasome substrates include damaged, misfolded and redundant proteins, and highly regulated members of critical signaling cascades $[4,5]$. This system is involved in regulating cell proliferation, differentiation, apoptosis, signal transduction, antigen presentation and inflammatory responses [6]. In malignant cells, UPP is 
frequently dysregulated. UPP has been implicated in tumor development and multidrug resistance. A growing body of evidence suggests that proteasome inhibition is more toxic to proliferating malignant cells than to normal cells $[7,8]$. The cytotoxic mechanisms include disruption of the cell cycle, triggering of apoptosis and ultimately tumor cell death [9]. Therefore, the UPP has emerged as one of the most attractive anti-cancer targets for drug design and development.

Small molecule inhibitors of the 20S proteasome are the most extensively studied proteasome inhibitors to date [10]. The first-in-class agent, bortezomib, was approved by FDA for the treatment of relapsed and/or refractory multiple myeloma (MM) or mantle cell lymphoma (MCL) $[11,12]$. It is a dipeptidyl boronic acid analogue that reversibly binds the catalytic $\beta$-subunit site of the $26 \mathrm{~S}$ proteasome in a concentration-dependent manner, thus predominantly inhibiting chymotrypsin-like (CT-L) and caspase-like (C-L) activity to a lesser degree [13]. Bortezomib has a wide range of molecular effects in $\mathrm{MM}$, including inhibition of NF- $\mathrm{BB}$ activation and induction of apoptosis [14, 15]. Specifically, bortezomib induced the intrinsic cell death pathway mediated by activation of caspase- 9 and caspase- 3 and extrinsic apoptotic signaling mediated by caspase- 8 and death receptors [16], as well as activation of heat shock proteins [17] and endoplasmic reticulum (ER) stress response pathways [18]. Bortezomib was also reported to influence the expression and inactivation of JNK [19] and DNA-dependent protein kinases [18]. Furthermore, bortezomib inhibited the interaction between MM cells and the bone marrow (BM) microenvironment, and blocked the transcription and secretion of associated MM cell growth factor [20]. Despite the potent antiMM activity of bortezomib, dose-related toxicities (e.g., peripheral neurotoxicity and thrombocytopenia) limited its long-term utility in clinical practices [21]. More importantly, all of the patients who initially responded to bortezomib eventually relapsed due to development of drug resistance [22]. At present, two other proteasome inhibitors, carfilzomib and ixazomib, were launched [23]. However, treatment with these drugs was associated with a number of toxicities, including cardiotoxicity, acute renal failure, thrombocytopenia, and neutropenia [23, 24].

In order to discover more effective proteasome inhibitors as anti-cancer drugs with broad therapeutic application and better safety profiles, different series of peptidyl boronic acid derivatives were previously designed, synthesized and biologically investigated by our group [25-28]. In this study, we used lead optimization by sulfur-based technology to identify a candidate NNU546, which was a novel $\mathrm{N}$-capped dipeptidyl boronic acid that immediately hydrolyzed to its biologically active form NNU219 upon exposure to aqueous solutions or plasma. NNU219 was able to selectively and irreversibly inhibit the chymotrypsin-like activity of the $20 \mathrm{~S}$ proteasome. We demonstrated that NNU219 inhibited tumor cell proliferation, induced the G2/M-phase arrest and apoptosis, downregulated NF- $\kappa B$ expression, and finally triggered an ER stress response in human MM cell lines and patient-derived cells at nanomolar concentrations. Importantly, NNU219 had a favorable cytotoxicity profile in normal human lymphocytes. Finally, three distinct human MM xenograft mouse models, including a cell-line-derived xenograft model (CDX), a patient-derived xenograft model (PDX) model and a SCID-rab model were used to evaluate the in vivo anti-cancer activities. Our results indicated that intravenous and oral administration of NNU219 and NNU546 were promising anti-MM candidates.

\section{RESULTS}

\section{NNU219 is identified as a candidate compound using sulfur-based technology}

To identify novel orally bioavailable proteasome inhibitors with improved efficacy and low toxicity from our previous work [25-28], we applied a unique sulfurbased technology to design and synthesize a diverse set of peptidyl boronic acid derivatives. The incorporation of methylthio group into the chemical scaffolds was aimed to improve metabolic stability, decrease toxicity and increase oral bioavailability by increasing drug exposure (area under the curve, AUC) and prolonging half-life $\left(\mathrm{t}_{1 / 2}\right)$. In the unique structural analogs, NNU219 was screened as the lead compound based on potency in proteasome inhibition and cell viability assays. Furthermore, the microsomal stabilities revealed that NNU219 had longer half-lives $\left(\mathrm{t}_{1 / 2}=17-32 \mathrm{~min}\right)$ and significantly shorter clearances $(\mathrm{CL}=43-83 \mathrm{~mL} / \mathrm{min} / \mathrm{kg})$ than bortezomib in rat, mice and human species (Supplementary Table 1). Pharmacokinetic studies of NNU219 in mice indicated a long half-life $(2.08 \pm 0.991$ h) and a large $\mathrm{AUC}_{0-\mathrm{t}}\left(2035 \mathrm{~h} \bullet \mathrm{ng}^{\bullet} \mathrm{mL}^{-1}\right)$ following intravenous administration. After oral administration, NNU546 was absorbed rapidly $\left(\mathrm{T}_{\max }=5 \mathrm{~min}\right)$ and displayed a long half-life $(2.41 \pm 0.420 \mathrm{~h})$ and good oral exposure (Supplementary Table 2).

\section{NNU219 selectively and potently inhibits proteasome activities in vitro}

The 20S proteolytic cores of constitutive proteasome (cCP) and immunoproteasome (iCP) have three different enzymatic activities, namely C-L, T-L and CT$\mathrm{L}$, which are encoded by the $\beta 1 \mathrm{c}, \beta 2 \mathrm{c}$ and $\beta 5 \mathrm{c}$, or $\beta 1 \mathrm{i}$, $\beta 2 \mathrm{i}$ and $\beta 5 \mathrm{i}$ subunits, respectively. To determine the 
selectivity of NNU219 towards proteasome catalytic subunits, we first evaluated the capacity of NNU219 to inhibit the activities of purified human erythrocytederived $\mathrm{cCP}$ and $\mathrm{iCP}$. The assays using the fluorogenic substrate indicated that NNU219 significantly inhibited the CT-L subunits of the constitutive proteasome $(\beta 5 \mathrm{c})$ and immunoproteasome ( $\beta 5 \mathrm{i})$ in a dose-dependent manner (Figure 1A; $p<0.01$ ) with less inhibition of the
A

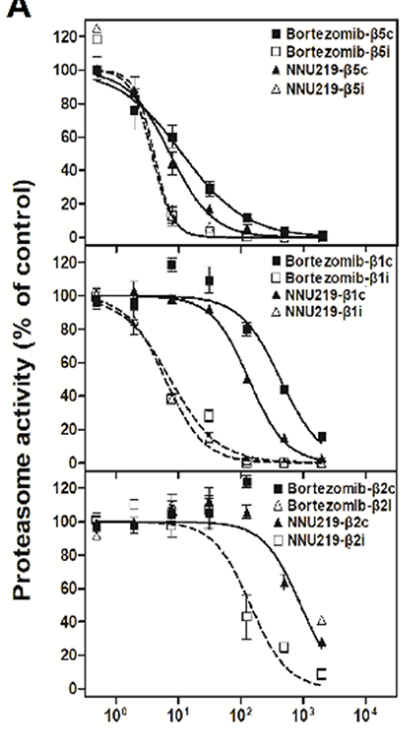

Proteasome inhibitor (nM)

B

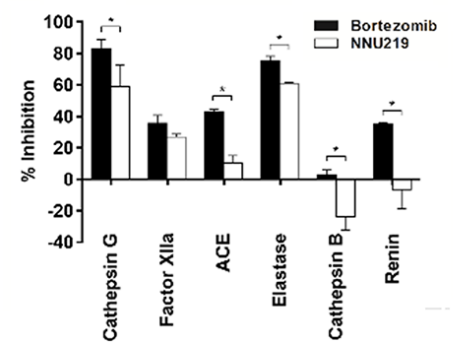

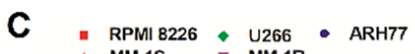

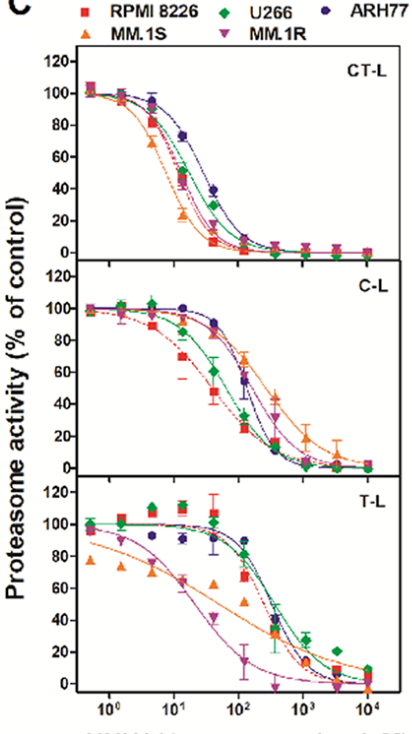

NNU219 concentration (nM)

\section{D}

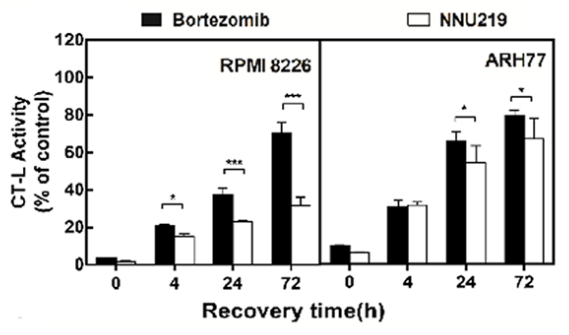

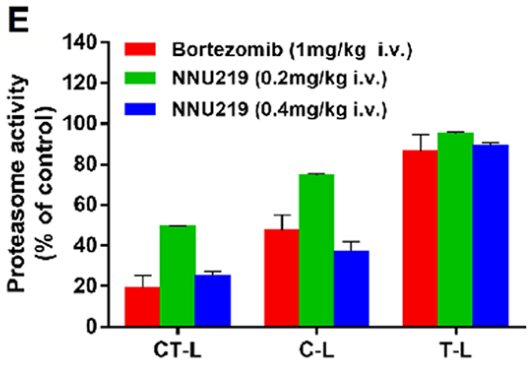
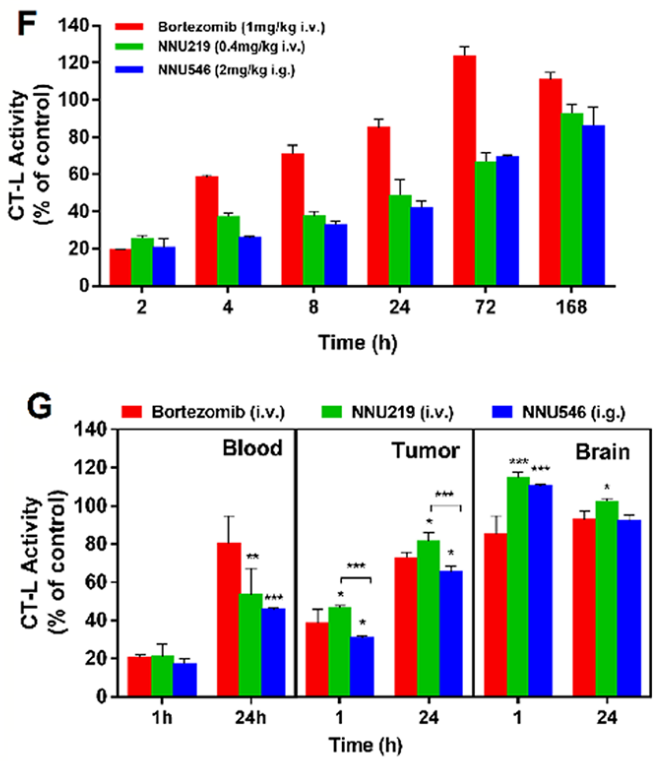

Figure 1. NNU219 and bortezomib differentially affect proteasome activities in vitro and in vivo. (A) In vitro effects of NNU219 or bortezomib on catalytic activities of the human constitutive proteasome $(\beta 1 c, \beta 2 c, \beta 5 c)$ and the immunoproteasome ( $\beta 1 \mathrm{i}, \beta 2 \mathrm{i}, \beta 5 \mathrm{i})$. Data from at least three independent measurements were normalized to DMSO-treated controls and were presented as residual activities \pm SD. (B) Inhibition of non-proteasomal proteases. NNU219 and bortezomib were tested at $10 \mu \mathrm{M}$ against a panel of purified serines (Cathepsin G, Factor XIla, Elastase), cysteine (Cathepsin B), aspartyl (Renin) and metallo (ACE) proteases. Percent inhibition was calculated based on the activities of compounds on protease subtracted with a substrate control without an enzyme. Data were presented as the mean inhibition \pm SD relative to DMSO-treated controls $(*, p<0.5)$. (C) Selectivity of NNU219 in the active sites of human MM cell lines. MM cells were treated with various concentrations of NNU219 for $1 \mathrm{~h}$ and cytosolic extracts were analyzed for CT-L, C-L and T-L proteasome activities. Results were represented as percent activities of proteasome in drug-treated vs. vehicle treated cells \pm SD. (D) Recovery of cellular proteasome activity following NNU219 or bortezomib treatment. Proteasome CT-L activity was determined in lysates prepared from RPMI 8226 (left panel) and ARH77 cells (right panel) at the indicated times following exposure to IC50 of NNU219 or bortezomib for $1 \mathrm{~h}$. Mean values from three measurements are presented as the percent activity relative to control-treated cells \pm SD $(*, p<0.5 ; * * *, p<0.001)$. (E) Proteasome active site selectivity of NNU219 in vivo. Mice $(\mathrm{n}=5)$ were treated with either NNU219 $(0.2 \mathrm{mg} / \mathrm{kg}$ or $0.4 \mathrm{mg} / \mathrm{kg}$ i.v.) or bortezomib (1 mg/kg i.v.) for 1 $\mathrm{h}$ and whole blood was analyzed for CT-L, C-L and T-L proteasome activities. (F) Inhibition and recovery of proteasome activity in vivo. Mice $(\mathrm{n}=5)$ were treated with NNU219 (0.4 mg/kg i.v.), NNU546 (2 mg/kg i.g.) and bortezomib (1 mg/kg i.v.) at the indicated time-points and blood samples were analyzed for CT-L proteasome activities. The data are represented as the percent inhibition compared with vehicle treated animals from two independent experiments. (G) ARH77 tumor-bearing mice were administered a single dose of NNU219 (0.4 mg/kg i.v.), NNU546 (2 mg/kg i.g.) or bortezomib (1 mg/kg i.v.); mice were euthanized at $1 \mathrm{~h}$ and $24 \mathrm{~h}$ time points after treatment. Heart, brain and tumor were harvested. Protein extracts were prepared and the proteasome catalytic activity was evaluated with CT-L subunit-specific fluorescent peptide substrates. Values were presented as the mean percent activity relative to vehicle \pm SD ( 3 mice per time-point). $p$ values presented for bortezomib vs NNU219 or NNU546 (*, $p<0.5 ; * *, p<0.01 ; * * *, p<0.001)$. SD, standard deviation; i.v. intravenous administration; i.g. intragastric administration; CT-L, chymotrypsin-like; C-L, caspase-like; T-L, trypsin-like; MM, multiple myeloma. 
T-L and C-L activities. These results indicated the selectivity of NNU219 to inhibit the CT-L activity of the proteasome. To further understand how NNU219 and MLN2238 (Ixazomib) are bound to $\beta 5$ subunit of proteasome, covalently theoretical docking was carried out and demonstrated that five strong hydrogen bonds were formed between these two small molecules with residues THR21, ALA49, GLY47 and ARG19 of $\beta 5$ subunit (Supplementary Figure 1). Furthermore, additional hydrophobic interaction existed between the methylthio group of NNU219 with residue ALA49 (Supplementary Figure 1A), which was beneficial to the binding of NNU219 with the $\beta 5$ subunit. However, there was no hydrophobic interaction between MLN2238 and ALA49 (Supplementary Figure 1B).

As bortezomib-induced peripheral neuropathy has been described to occur via a proteasome-independent mechanism in vitro and bortezomib inhibited several nonproteasomal targets in vitro and in vivo [29], the binding of NNU219 with possible non-proteasomal targets was also evaluated. NNU219 had relatively weak or no inhibitory activities against most of the nonproteasomal proteases except cathepsin $\mathrm{G}$ and elastase, with $50 \%$ inhibition at $10 \mu \mathrm{M}$ (Figure 1B), which showed that NNU219 was less potent to nonproteasomal proteins than bortezomib (Figure 1B; all $p$ values $<0.05$ but Factor XIIa was not significant).

It has been previously reported that the binding of proteasome inhibitors to isolated enzymes was different in the living cells [30] Therefore, it was of interest to determine the ability of NNU219 to inhibit the three subunits in MM cells. Five MM cell lines were treated with 0-10 $\mu \mathrm{M}$ NNU219 or bortezomib for $1 \mathrm{~h}$ and then analyzed for CT-L, C-L and T-L proteasome activities using the Proteasome-Glo cell-based assay. Incubation of MM cells with NNU219 resulted in a dose-dependent inhibition of catalytic activities of the three subunits with the activity of CT-L was inhibited to the greatest extent, which was similar to the results obtained with purified 20S proteasome (Figure 1C; $p<0.05$ ). The C-L and T-L activities in the cells were inhibited to a greater extent than the inhibitory effect on the isolated enzyme (Figure 1C; $p<0.05$ ). Bortezomib also exerted similar effects (Supplementary Figure 2). Although NNU219 covalently interacted with the proteasome, the dissociation of the NNU219-proteasome complex could be different from that of bortezomib. Therefore, the effect of NNU219 on the recovery of proteasome activity in RPMI 8226 and ARH77 cells was then investigated. The MM cells were treated with NNU219 for $1 \mathrm{~h}$, allowed to recover in drug-free media and then CT-L activity was monitored. Our results showed that the proteasome activity in ARH77 cells treated with NNU219 recovered to $67 \%$ after $72 \mathrm{~h}$, whereas the activity in RPMI 8226 cells recovered only to $31 \%$. The proteasome activity recovery rates in NNU219-treated cells were slower than those obtained after bortezomib treatment (Figure 1D; $p<0.001$ and $p<0.05$ in ARH77 cells and RPMI 8226 cells, respectively). Taken together, these in vitro assays indicated that NNU219 is a potent and selective inhibitor of the $20 \mathrm{~S}$ proteasome.

\section{NNU219 displays good pharmacodynamic profiles and oral bioactivity in vivo}

The inhibition of 20S proteasome activity induced by NNU219 and bortezomib was further investigated in vivo. First, the selectivity of NNU219 and bortezomib to the active sites of the proteasome was investigated. Mice were intravenously administered with different concentrations of NNU219 (0.2 and $0.4 \mathrm{mg} / \mathrm{kg})$ or bortezomib $(1 \mathrm{mg} / \mathrm{kg})$. Whole blood was collected after $1 \mathrm{~h}$ and the CT-L, C-L and T-L activities were analyzed. We found that NNU219 decreased the CT-L and C-L activities in a dose-dependent manner. Notably, while both bortezomib $(1 \mathrm{mg} / \mathrm{kg})$ and NNU219 $(0.4 \mathrm{mg} / \mathrm{kg})$ similarly inhibited CT-L $(p<0.001)$, the dosage of NNU219 used to reach this effect was substantially lower (Figure 1E). Furthermore, at doses that induced $>70 \%$ inhibition of CT-L activity in the blood, the two compounds were able to inhibit the activity of C-L $(p<$ $0.01)$. However, the two compounds did not inhibit the T-L activity (Figure 1E). These results indicated that NNU219 could selectively inhibit proteasome activities in vivo and were consistent with the results obtained in cell lines shown in Figure 1C.

Given that NNU219 and NNU546 had good microsomal stabilities and pharmacokinetic properties, their pharmacodynamic profiles were then investigated in vivo with intravenous and oral administrations. The recovery of proteasome activity in whole blood was determined at different time points after intravenous injection of NNU219 and bortezomib or oral administration of NNU546. The activities of CT-L proteasomes were reduced by up to $80 \%$ after $2 \mathrm{~h}$ following administration of the compounds (Figure 1F; $p<0.001$ ). Proteasome activities began to recover after $4 \mathrm{~h}$, almost recovered to baseline after $24 \mathrm{~h}$ and completely recovered after $72 \mathrm{~h}$ following administration of bortezomib. In contrast, much slower recovery of proteasome activities was observed following intravenous administration of NNU219 compared with bortezomib $(p<0.001)$. Furthermore, oral administration of NNU546 triggered a greater and more prolonged dose-related inhibition of proteasome activities in the whole blood $(p<0.001)$.

To evaluate the proteasome activities in tumors and normal host tissues, ARH77 MM tumor-bearing mice 
were intravenously injected with a single dose of NNU219 $(0.4 \mathrm{mg} / \mathrm{kg})$, bortezomib $(1 \mathrm{mg} / \mathrm{kg})$ or orally administered with NNU546 $(2 \mathrm{mg} / \mathrm{kg})$ and euthanized after 1 or $24 \mathrm{~h}$ post-administration based on the preliminary dose-exploratory experiment. Heart, liver, spleen, lung, kidney, brain and tumor were collected and examined for CT-L proteasome activities. The results showed that proteasome activities were significantly reduced in tumor specimens as well as blood and various normal mouse tissues (except the brain) within $1 \mathrm{~h}$ following administration of all three tested compounds (Figure 1G and Supplementary Figure 3; $p<0.01$ ). Nevertheless, at $24 \mathrm{~h}$ post injection, proteasome activities gradually recovered as compared to vehicle (Figure 1G and Supplementary Figure 3). Similar proteasome activity recovery patterns were shown for bortezomib, NNU219 and NNU546. Importantly, no significant reduction in proteasome activity was detected in brain tissue at any time point for either compound (Figure 1G), which indicated that they did not cross the blood-brain barrier (BBB). Notably, oral administration of NNU546 showed much greater proteasome inhibition in blood and tumor samples compared to NNU219 and bortezomib (Figure 1G). Taken together, these data indicate that both NNU219 and NNU546 are bioavailable and selective compounds with favorable pharmacodynamic responses.

\section{NNU219 inhibits the proliferation of a variety of tumor cells in vitro}

To investigate the difference in antiproliferative activity between NNU219 and bortezomib in hematological and solid tumor cell lines, 5 hematological cell lines (MM.1S, MM.1R, U266, RPMI 8226, and ARH77) and 5 cell lines established from solid malignancies (HCC1937, MDA-MB-231, A549, SCG7901, and $\mathrm{BxPC}-3$ ) were treated with various concentrations of the test compounds for 24-72 $\mathrm{h}$ and cell viability was measured using CCK-8 assay. Most previous studies evaluated the effect of proteasome inhibition on cultured cells using extended treatment periods (24-72 h), which in fact did not reflect the rapid clearance of the drug from plasma [30]. In this manuscript, the cytotoxic effects of NNU219 and bortezomib were evaluated on a panel of tumor cell lines treated for $1 \mathrm{~h}$ and followed by $72 \mathrm{~h}$ washout period. The results suggested that the two compounds had greater antiproliferative effects against the hematological tumor cell lines than the solid ones (Figure 2A). Prolonged treatment with NNU219 and bortezomib for $72 \mathrm{~h}$ without washout displayed similar trend but resulted in greater cytotoxicity (Figure 2B; $p<0.001$ for all cell lines). Suppression of recovery of proteasome activity might be responsible for the increased cytotoxicity observed with extended treatment time.
Finally, to determine the effect of NNU219 on cells isolated from patients with $\mathrm{MM}, \mathrm{CD} 138^{+}$cells were purified from $\mathrm{BM}$ aspiration of $5 \mathrm{MM}$ patients who were newly diagnosed (patients no. 2, 3 and 4) or exposed to multiple therapeutic protocols (patients no. 1 and 5 had previously received bortezomib, dexamethasone, or lenalidomide) and were treated with NNU219 for $24 \mathrm{~h}$. While direct comparison between NNU219 and bortezomib in primary cultures was precluded by the relatively small number of $\mathrm{CD} 138^{+}$ cells isolated from these patients, a significant dosedependent decrease in viability of the cells from all MM patients was observed after NNU219 treatment (Figure $2 \mathrm{C} ; p<0.001$ for all patient samples). Particularly, prolongation of NNU219 treatment from 36 to $48 \mathrm{~h}$ resulted in greater inhibition of cell viability (Figure $2 \mathrm{D} ; p<0.001)$. These results showed that the ability of NNU219 to trigger cytotoxicity was not only limited to tumor-derived MM cell lines and could be extended to primary MM cells from refractory patients including those exposed to therapy regimens with bortezomib, dexamethasone or lenalidomide. Importantly, NNU219 exhibited a favorable cytotoxicity profile toward BMderived stromal cells (BMSCs)/CD138- cells (Figure 2E). In addition, NNU219 did not significantly affect the viability of normal PBMCs from healthy volunteers, which retained $>80 \%$ viability at $1,000 \mathrm{nM}$ (Figure $2 \mathrm{~F}$ ), suggesting specific and selective anti-MM activity and a favorable therapeutic index for NNU219.

\section{NNU219 induces cell cycle arrest and apoptosis in MM cells}

To determine the possible mechanisms responsible for the cytotoxic effects of NNU219, apoptosis and cell cycle analysis were performed after NNU219 treatment. MM cells were incubated with 0-20 nM NNU219 for 24-48 $\mathrm{h}$ and the cell cycle distribution was examined by flow cytometry. Compared with the untreated cells, the proportions of cells in G0/G1 and G2/M phase in the treatment groups were significantly reduced and increased, respectively (Figure $3 \mathrm{~A} ; p<0.05$ ). Apoptosis was then examined after Annexin V-FITC/PI fluorescence staining. Flow cytometric analysis revealed a significant increase in the apoptotic cell population after treatment with 10 and $20 \mathrm{nM}$ of NNU219 for $24 \mathrm{~h}$ compared with the untreated cells (Figure 3B; $p<0.001$ ). Incubation of MM cells with NNU219 for $48 \mathrm{~h}$ led to higher apoptosis than that seen at $24 \mathrm{~h}$. (Figure 3B). These results indicated that NNU219 was capable of inducing apoptosis in MM cells in a time- and dose-dependent manner.

To further confirm the molecular mechanism by which NNU219 induces cytotoxic effects in cells, the expression of cell cycle-regulatory proteins and 
A

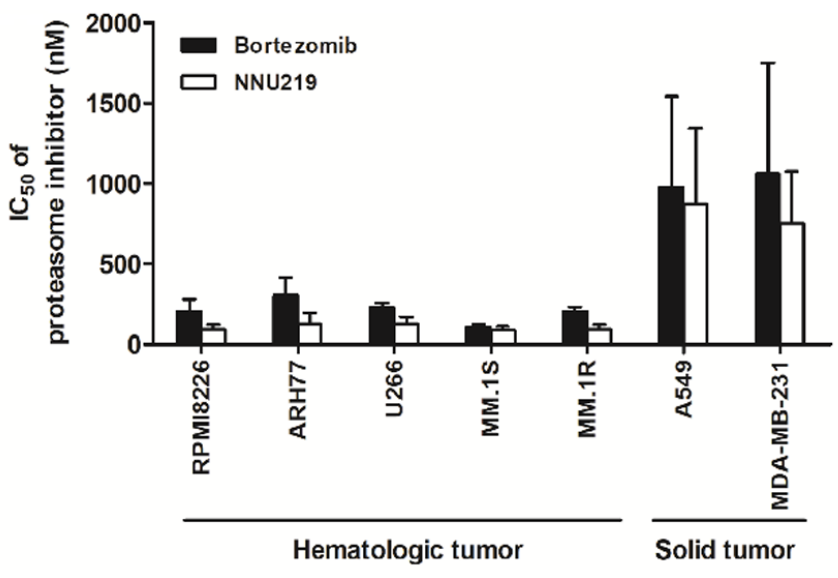

C

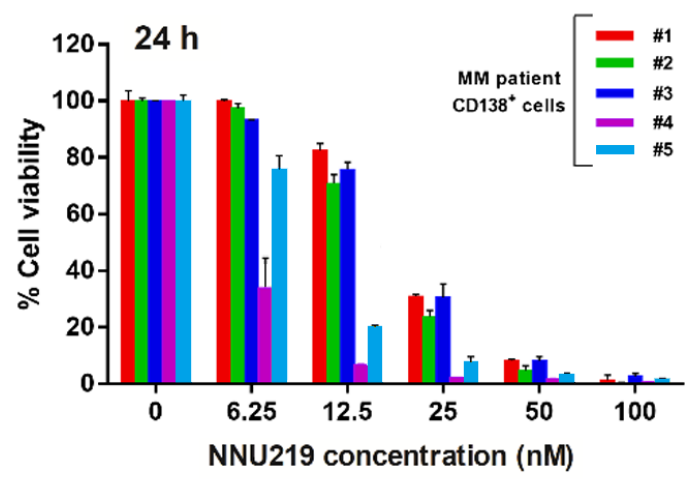

E

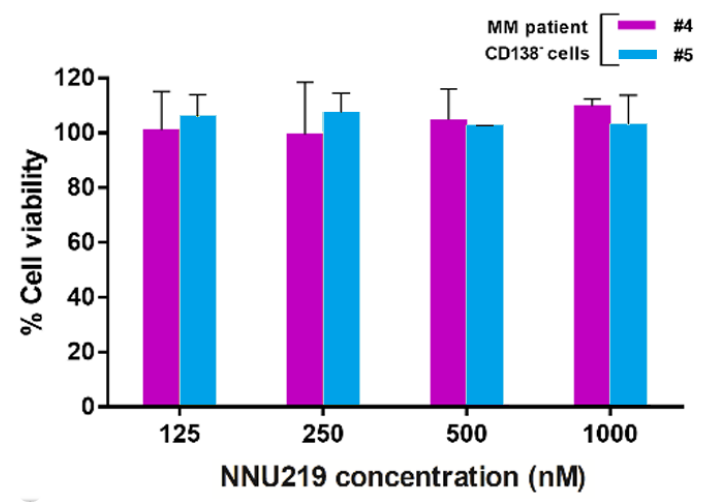

B

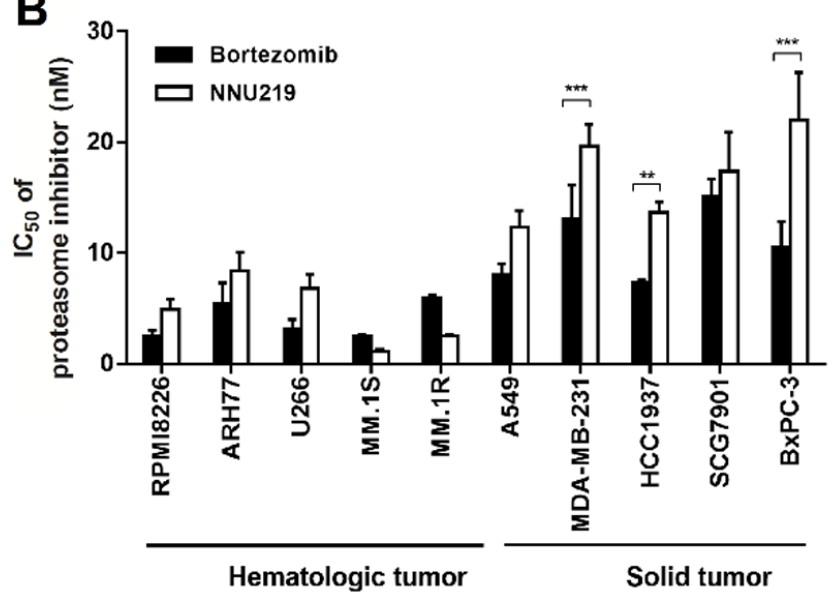

D

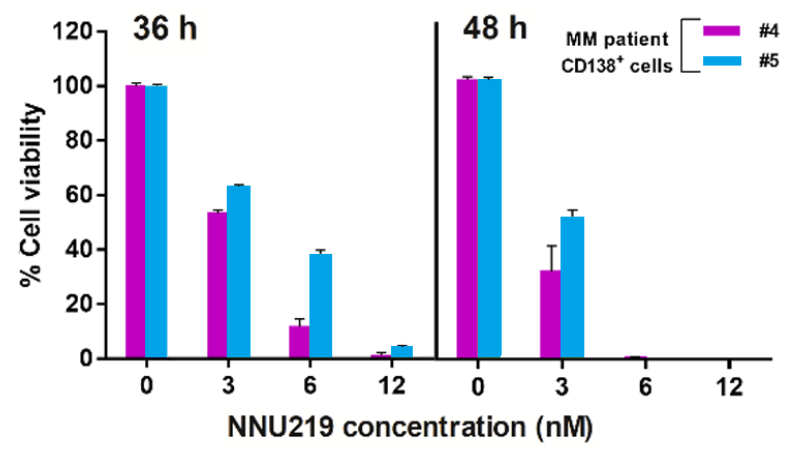

$\mathbf{F}$

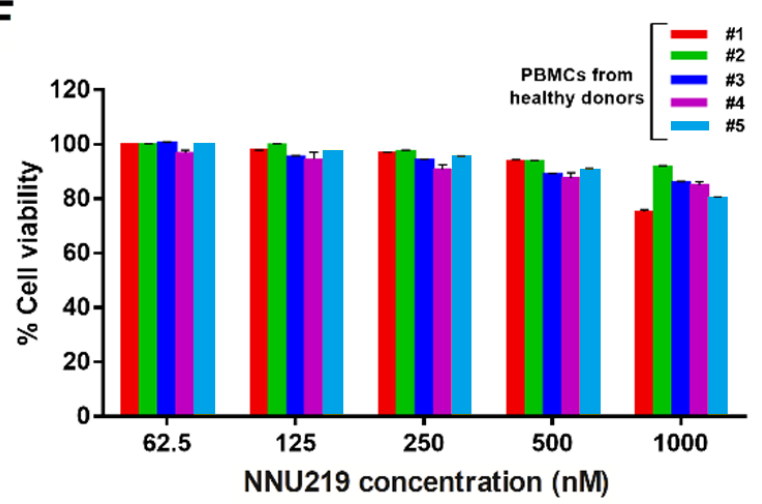

Figure 2. Cytotoxicity profiles of NNU219 and bortezomib against tumor cell lines, primary myeloma cells and human PBMC. (A, B) Hematological and solid tumor cell lines were treated with NNU219 and bortezomib. Cell viability was measured with CCK8 reagent either after $1 \mathrm{~h}$ of compound treatment followed by $72 \mathrm{~h}$ washout period (A) or continuous compound treatment for $72 \mathrm{~h}$ (B). The $\mathrm{IC}_{50}$ value as a measure the cytotoxic effects of tested compounds. Values were the mean \pm SD from 3 determinations $\left({ }^{* *}, p<\right.$ $\left.0.01 ;{ }^{* *}, p<0.001\right)$. (C) Purified patient MM cells $\left(\mathrm{CD} 138^{+}\right)$were treated with increasing concentrations of NNU219. The cytotoxic effects of NNU219 were determined by bioluminescent measurement of cellular ATP using Cell Titer-Glo reagent after $24 \mathrm{~h}$ treatment. Results were expressed as \% cell viability over DMSO control. Data were presented as mean \pm SD of triplicate samples $(p<0.001$ for all patient samples). (D) CD138+ cells from MM patient no. 4 (newly diagnosed) and no. 5 (refractory) were treated with NNU219 for $36 \mathrm{~h}$ and $48 \mathrm{~h}$, respectively. Data were presented as mean \pm SD of triplicate samples $(p<0.001)$. (E) CD138 cells from MM patient no. 4 and no. 5 were treated with NNU219 for $24 \mathrm{~h}$. Data were shown as mean \pm SD of triplicate samples. (F) PBMCs from healthy volunteers were treated with increasing concentrations of NNU219 and then analyzed for viability using Cell Titer-Glo assay. Data were shown as mean \pm SD of triplicate samples. 

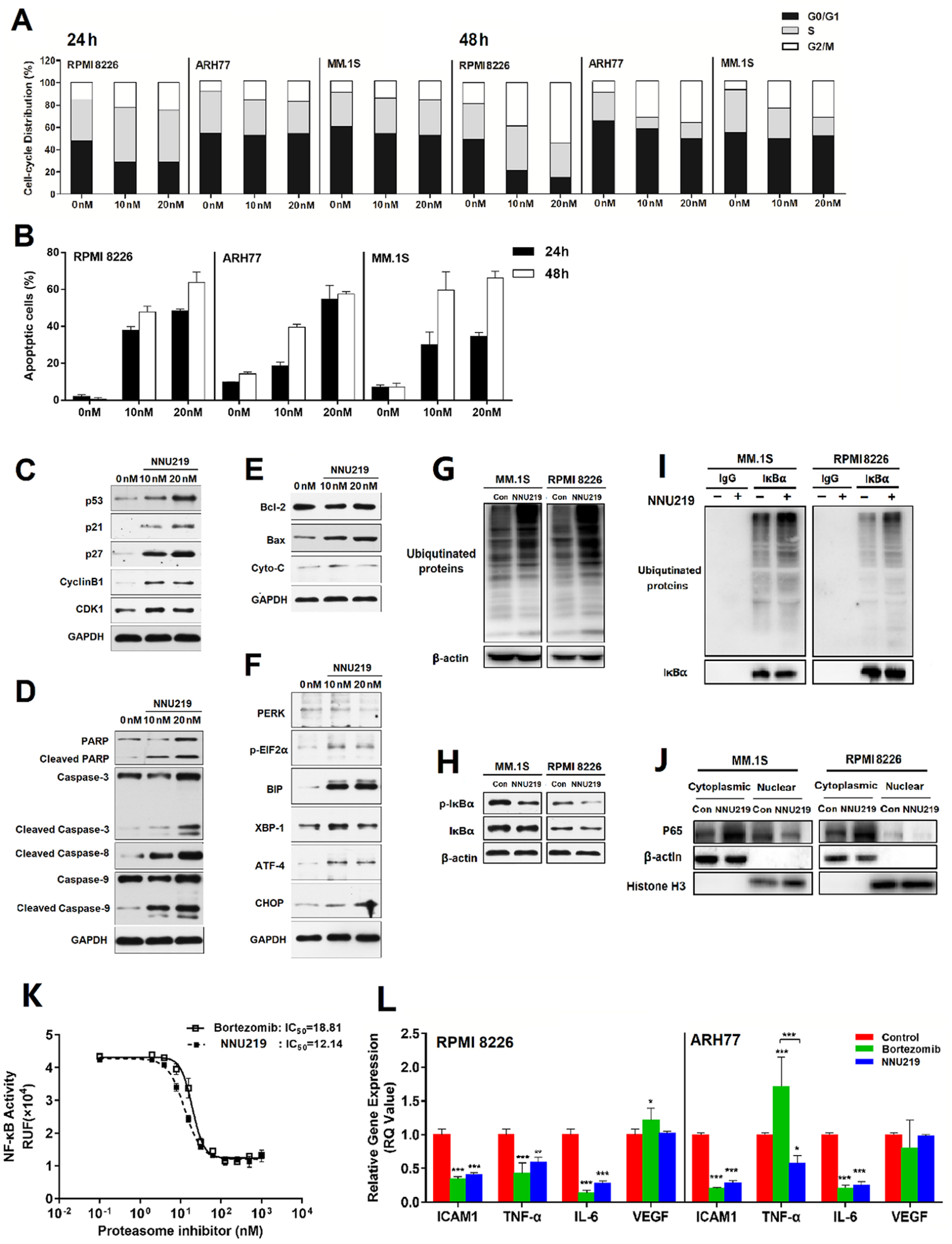

Figure 3. NNU219 induces apoptotic signal transduction and modulates the NF-KB signaling pathway in human MM cell lines. (A) The MM cell lines RPMI 8226, ARH77 and MM.1S were incubated with DMSO or NNU219 (10 and $20 \mathrm{nM}$ ) for 24 or $48 \mathrm{~h}$, respectively. Cells 
were fixed with ethanol and stained with propidium iodide, then DNA content was determined by flow cytometry ( $p<0.05$ for all cell lines). (B) The MM cell lines RPMI 8226, ARH77 and MM.1S were incubated with DMSO or NNU219 (10 and $20 \mathrm{nM}$ ) for 24 or $48 \mathrm{~h}$ and induction of apoptosis was determined after annexin V-FITC/propidium iodide staining by flow cytometry. Data were presented as mean \pm SD of three independent experiments ( $p<0.001$ for all cell lines). (C-F) RPMI 8226 cells were incubated with NNU219 (10 and $20 \mathrm{nM}$ ) for 24 h. After the incubation, cells were lysed and directly subjected to SDS-PAGE, transferred to membranes and blotted with indicated antibodies. GAPDH immunoblotting was included for protein loading control. Blots in the figures were representatives of three independent experiments. (G) MM1.S and RPMI 8226 cell lines were treated with $10 \mathrm{nM}$ of NNU219 for $24 \mathrm{~h}$. The stimulated cells were lysed and $20 \mu \mathrm{g}$ of protein was processed for ubiquitin immunoblotting. (H) MM1.S and RPMI 8226 cells were treated with DMSO, 10 nM of NNU219 for 24 h and immunoblotted using anti-IKB $\alpha$ and anti-phospho-IKB $\alpha$ antibodies. (I) MM1.S and RPMI 8226 cell lines were treated with 10 nM of NNU219 for $24 \mathrm{~h}$. Correlative proteins were immunoprecipitated from $1 \mathrm{mg}$ of MM cell lysate using IKB $\alpha$ or IgG antibody (rabbit) and coupled to protein A/G agarose beads. The beads were washed by IP buffer and processed by immunoblotting for ubiquitin, p65 or IKB $\alpha$. (J) MM1.S and RPMI 8226 cell line was treated with $10 \mathrm{nM}$ of NNU219 for $24 \mathrm{~h}$. Nuclear and cytoplasmic protein fractions were separated from the total lysate and analyzed for $\mathrm{p65}, \beta$-actin and histone antibodies by western blot analysis. Blots in the figure were representative of three independent experiments. (K) The transfected NF-KB/Luciferase 293T cells were incubated with increasing concentrations of NNU219 or bortezomib for $6 \mathrm{~h}$. Cells were then stimulated with $10 \mathrm{ng} / \mathrm{ml}$ of TNF- $\alpha$ for another $18 \mathrm{~h}$. The activity of expressed luciferase was determined by using the Dual-Luciferase Reporter Assay System. (L) RPMI 8226 or ARH77 cell lines were treated with DMSO, IC 50 of NNU219 or bortezomib for $12 \mathrm{~h}$ and harvested. Total RNA was isolated and subjected to qRT-PCR. The gene expression level of ICAM1, TNF- $\alpha$, IL6 and VEGF was normalized to GAPDH using the $2^{-\triangle \Delta C T}$ method. In above experiments, the control group was incubated with the same concentration of DMSO in normal culture medium. Values were expressed as mean \pm SD of triplicate samples of three independent experiments (*, $p<0.05 ;{ }^{* *}, p<0.01 ;{ }^{* *}, p<0.001$ ). IL, interleukin; TNF, tumor necrosis factor; ICAM, intercellular adhesion molecule; VEGF, vascular endothelial growth factor; $\mathrm{p}-\mathrm{I} \mathrm{KB} \alpha$, phosphorylated inhibitor of NF-kB; Con, control; MM, multiple myeloma.

activation of caspases were examined by western blot analysis. The results showed that treatment of MM cells with NNU219 resulted in increased expressions of cyclin B1 and CDK1. Furthermore, p21, p27 and p53 were also upregulated (Figure 3C). In addition, NNU219 induced caspase-3, caspase-8, caspase-9 and PARP cleavage in dose-dependent manners. These observations indicated that NNU219 triggered mitochondria-dependent and -independent apoptotic cell death signaling pathways, leading to activation of the apoptosis in MM cells (Figure 3D). To investigate the effect of NNU219 on the mitochondrial protein family, the expression of Bax, Bcl-2 and cytochrome-C was determined following incubation of $\mathrm{MM}$ cells with increasing concentrations of NNU219. Although expression of the anti-apoptotic protein Bcl-2 was not changed, the levels of Bax and cytochrome-C proteins were substantially induced (Figure 3E).

Accumulation of excessive or misfolded proteins in the ER triggers the unfolded protein response (UPR) [23]. Previous studies indicated that induction of ER stress in MM cells was an effective anti-cancer strategy and attributed to one of the mechanisms of cytotoxicity in MM cells [23, 31]. Therefore, the role of NNU219 in regulating the expression of UPR components in MM cells was then investigated, revealing that NNU219 caused activation of the PERK-mediated ER stress signal. As shown in Figure 3F, NNU219 induced elevation of BIP and XBP-1 together with triggered phosphorylation of EIF2 $\alpha$, further leading to expression of transcription factor ATF-4 and pro-apoptotic signaling molecule CHOP. These results indicate that NNU219 induces cell death via induction of UPRdependent ER stress.
As inhibition of proteasome activity leads to blockade of the proteasome-dependent polyubiquitination protein degradation pathway and thereby triggers apoptosis [22], MM cells were treated with NNU219 and then the protein lysates were subjected to western blot analysis with a ubiquitin-specific monoclonal antibody (Figure 3G). The results revealed that incubation with NNU219 resulted in marked accumulation of ubiquitinated proteins as a result of inhibited proteasome function, which may induce activation of multiple pro-apoptotic signaling axes [22, 30].

\section{NNU219 targets NF-кB activity}

It was previously reported that one of the major mechanisms underlying the treatment of $\mathrm{MM}$ by proteasome inhibitors is blockade of the NF- $\kappa \mathrm{B}$ signaling $[6,32]$. NF- $\kappa \mathrm{B}$ mediated immune and inflammatory responses and was thought to be associated with proliferation, survival and drug resistance in MM cells [23]. Therefore, as a next step we examined whether NNU219 could reduce NF- $\mathrm{NB}$ activity in MM cells. The results demonstrated that NNU219 substantially blocked the phosphorylation of $\mathrm{I} \kappa \mathrm{B} \alpha$ (Figure $3 \mathrm{H}$ ). Inhibition of $\mathrm{I} \kappa \mathrm{B} \alpha$ phosphorylation indicates reduced degradation of this protein and subsequently more binding to NF- $\kappa \mathrm{B}$, thereby blocking the NF- $\kappa B$ signaling pathway. Given the effects of NNU219 on NF- $\kappa B$ pathway regulation, the role of NNU219 in the ubiquitination of $\mathrm{I} \kappa \mathrm{B} \alpha$ was also investigated. As presented in Figure 3I, NNU219 promoted the accumulation of $\mathrm{I} \kappa \mathrm{B} \alpha$ ubiquitination in MM cells. Moreover, immunoblotting analysis of cytoplasmic and nuclear extracts demonstrated that nuclear p65 level was substantially reduced by NNU219 
(Figure 3J). Next, NF-kB/Luc 293T cells were used to detect NF- $\kappa \mathrm{B}$ activity. The 293T cell line was transfected with luciferase reporter vector under the control of NF- $\kappa B$-responsive element. NF- $\kappa B$ was rapidly activated by stimulation with $\mathrm{TNF}-\alpha$, inducing an increase in the luciferase activity. However, pretreatment of NF- $\kappa \mathrm{B} / \mathrm{Luc} 293 \mathrm{~T}$ cells with NNU219 led to a significant reduction of luciferase activity in a timedependent manner (Figure 3K, $p<0.001$ ). Several studies previously suggested that adhesion of MM cells to BMSCs triggered NF- $\kappa \mathrm{B}$-mediated transcription and cytokine secretion, which was associated with growth and survival of MM cells, including the production of cytokines (IL- 6, IL-1- $\beta$, TNF- $\alpha$ ), cell adhesion molecules (ICAM), vascular cell adhesion molecule (VCAM) and the proangiogenic factor (VEGF)c. Therefore, the expression levels of these genes were tested in RPMI 8226 and ARH77 cells after either NNU219 or bortezomib treatment. The results showed that NNU219 inhibited the expression of the cytokines, while no significant changes in the expression of VEGF were observed. However, treatment with bortezomib resulted in upregulation of VEGF expression in RPMI 8226 cells and of TNF- $\alpha$ in ARH77 cell line (Figure $3 \mathrm{~L})$. Collectively, these results indicated that NNU219 targets NF- $\kappa \mathrm{B}$ and related cytokine secretion similarly to, or even better than bortezomib, albeit in lower concentration.

\section{NNU219 inhibits tumor growth in human MM cell xenograft mouse models}

NNU219 induced MM cells apoptosis in vitro and had good pharmacodynamic profiles in vivo. Therefore, we next evaluated the anti-tumor efficacy of NNU219 using 3 different xenograft mouse models (Figure 4). Both NNU219 and NNU546 dosing schedules were tolerated in the tumor-bearing animals, resulting in weight loss of $<10 \%$ (data not shown). The bortezomib and ixazomib dosing schedules were determined to be the maximum tolerated dose (MTD) in this mouse strain $[3,12]$. First, ARH77 tumor-bearing mice were intravenously treated with bortezomib $(1 \mathrm{mg} / \mathrm{kg}, \mathrm{BIW})$, NNU219 (0.4 mg/kg, BIW) or vehicle (BIW), respectively. As shown in Figure $4 \mathrm{~A}$ and $4 \mathrm{~B}$, administration of either NNU219 or bortezomib significantly reduced the tumor burden compared with vehicle group $(p<0.05)$. Furthermore, NNU219 exhibited a relatively higher efficacy than bortezomib in reducing the tumor volume (75.3 vs. $24.9 \%)$. For oral administrations (i.g.), the anti-tumor effect of NNU546 (1 $\mathrm{mg} / \mathrm{kg}$, QD or $2 \mathrm{mg} / \mathrm{kg}$, QOD) was investigated along with ixazomib $(5 \mathrm{mg} / \mathrm{kg}, \mathrm{BIW})$ as the positive control. The daily dosing schedules of NNU546 was more intensive than the preclinical dosing schedule for ixazomib, preventing full recovery of proteasome activity between doses. Animals were continuously treated for 3 weeks and the tumor volume was measured. A dose-dependent tumor growth inhibition was achieved by NNU546 with 1 and $2 \mathrm{mg} / \mathrm{kg}$, leading to reduction in tumor volume by $74.6 \%$ and $83.9 \%$, respectively (Figure 4C, 4D; $p<0.05$ ).

Treatment with ixazomib also reduced tumor progression by $36.3 \%$, but less than the effect of NNU546. Importantly, no weight loss, diarrhea, hair loss, or neurological symptoms were observed in NNU546-treated mice, even if mice were treated daily with $1 \mathrm{mg} / \mathrm{kg}$ dose (data not shown), which may indicate that NNU546 has greater tolerability and less toxic in vivo.

In addition, paraffin-embedded sections of tumors from xenograft mice were subjected to immunofluorescence (IF) staining. The results are shown in Figure 4E. TUNEL positive cells (green color, an indication of cell apoptosis) were increased in tumor sections of NNU546-treated mice compared with those of the vehicle-treated counterparts. In parallel, the proliferation marker Ki-67 (red color) was also significantly decreased. These results confirmed the potent apoptosis-inducing effect of NNU546 in MM cells in vivo, which was consistent with the in vitro data. MM cell growth was previously reported to be associated with angiogenesis $[22,31]$. Therefore, the anti-angiogenic activity of NNU546 was then evaluated by using two distinct markers of angiogenesis, CD31 and $\alpha$-SMA. As shown in Figure 4E, treatment with NNU546 reduced the density of CD31 (green color), $\alpha$ SMA (red color) and the number of endothelial cells and surrounding pericytes in tumor tissues, further confirming the in vivo anti-tumor and anti-angiogenesis activities of NNU546 in MM.

To further evaluate the therapeutic efficacy of NNU546, patient-derived primary human MM xenografts implanted in NCG mice were established. Compared with the control group, treatment with NNU546 (1 mg/kg, i.g., QD) significantly inhibited the tumor growth (46.1\% of reduction of tumor volume) (Figure 5A-5C; $p<0.05$ ). In comparison, ixazomib (5 $\mathrm{mg} / \mathrm{kg}$, i.g., BIW) had a less potent effect to induce tumor regression $(30.5 \%$ of reduction of tumor volume). Once the administrations were completed, the mice were euthanized and tumor sections were examined by $H \& E$ staining. We found that large areas of necrosis with abundant nuclear debris appeared in xenograft mice treated with NNU546. In addition, NNU546 markedly reduced the expression of Ki67, the density of CD31 and $\alpha$-SMA in blood vessels and increased the apoptosis of MM cells detected by IF assay (Figure 5D). 
A

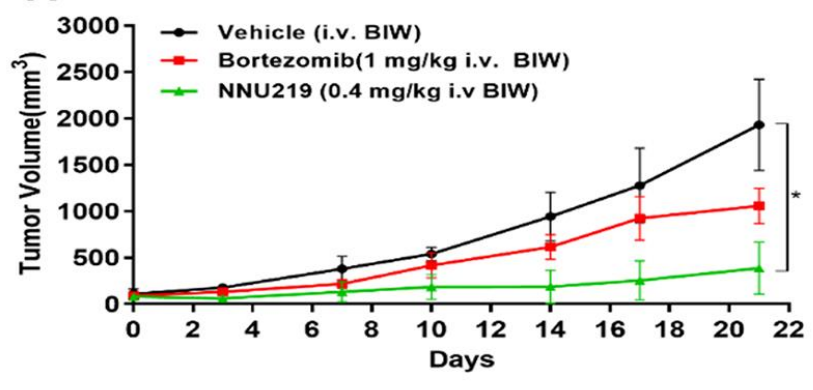

C

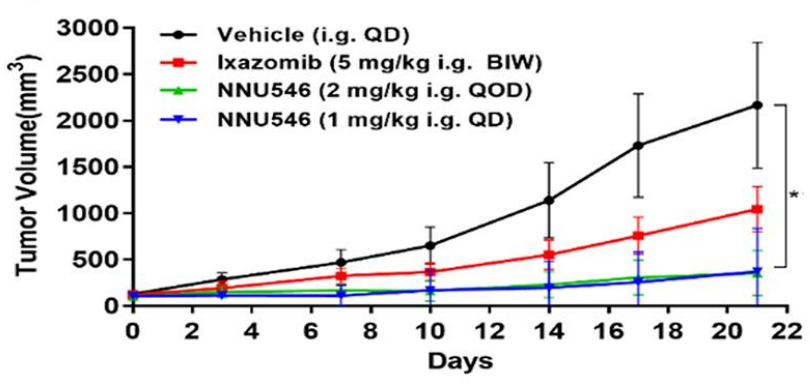

B

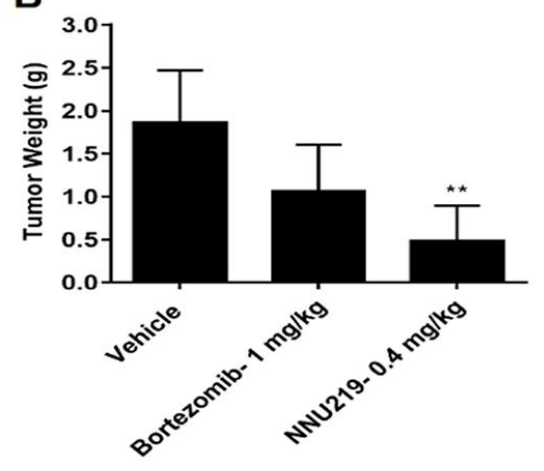

D

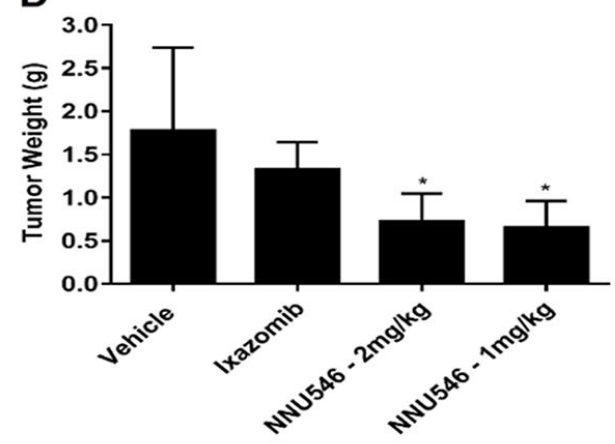

E
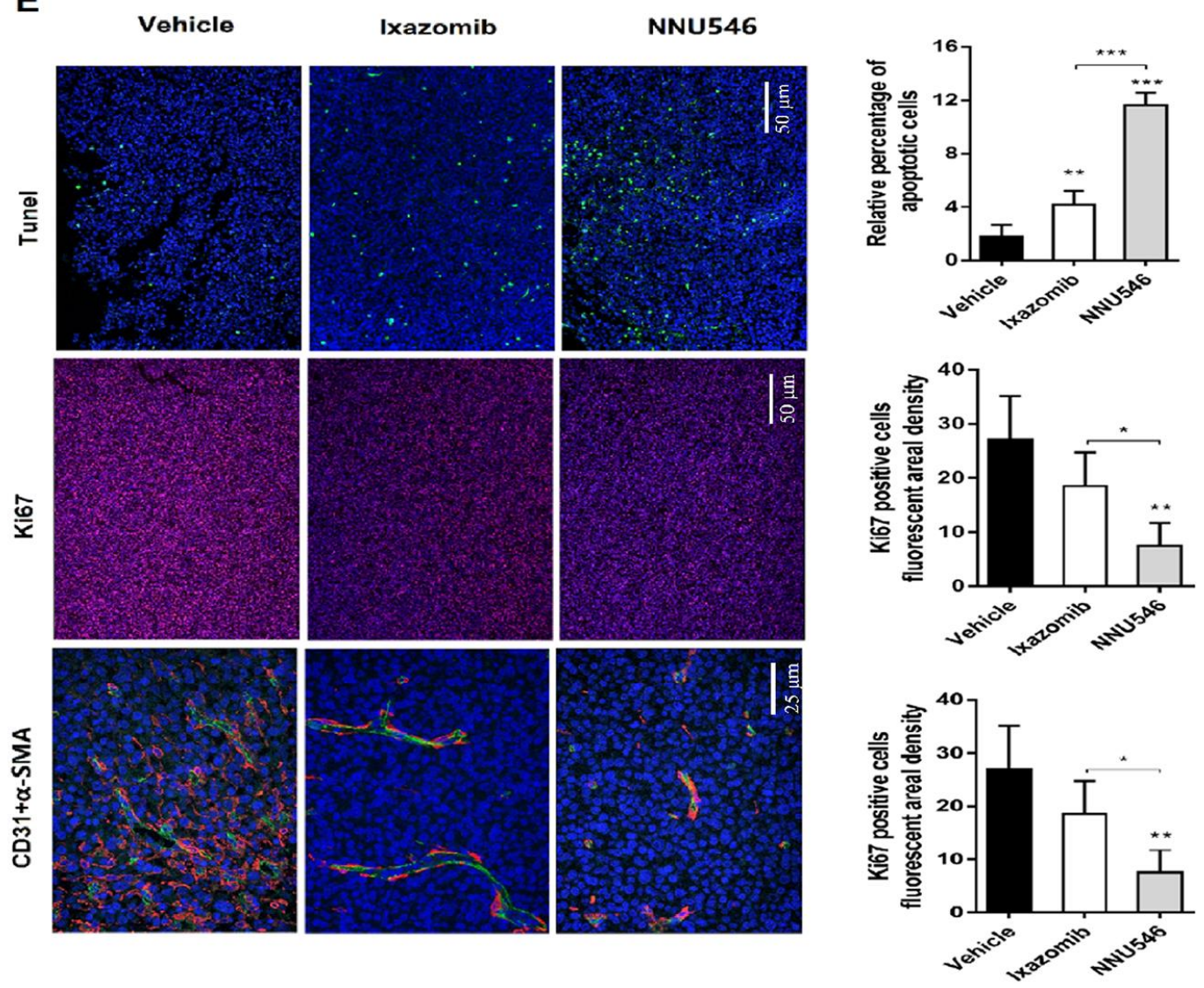

Figure 4. Effects of NNU219, NNU546, bortezomib and ixazomib on the growth of ARH77 xenograft model established in nude mice. (A) $5 \times 10^{6}$ of ARH77 cells were subcutaneously inoculated into the right flank of nude mice. When the mean tumor volume 
reached $100-150 \mathrm{~mm}^{3}$, mice were randomized into vehicle group (1\% DMSO and 5\% HPBCD) and treatment group (bortezomib, $1 \mathrm{mg} / \mathrm{kg}$ or NNU219, $0.4 \mathrm{mg} / \mathrm{kg}$ ), intravenously administered twice weekly for 3 weeks. The measurement was performed using a caliper. Data were presented as mean tumor volume $\pm \mathrm{SD}(\mathrm{n}=5 ; *, p<0.05)$. (B) Average tumor weight of mice in the vehicle and treatment groups. Data were

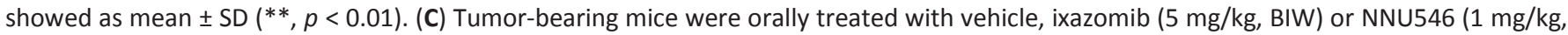
$\mathrm{QD}$ or $2 \mathrm{mg} / \mathrm{kg}, \mathrm{QOD}$ ) schedule for 3 weeks. Data were presented as mean tumor volume $\pm \mathrm{SD}\left(\mathrm{n}=5 ;{ }^{*}, p<0.05\right)$. (D) Average tumor weight of mice in the vehicle and treatment groups. Data were showed as mean $\pm \mathrm{SD}(*, p<0.05)$. (E) Left, tumor sections of untreated, Ixazomib or NNU546 treated mice were subjected to immunostaining for apoptosis (TUNEL, green color), proliferation marker Ki-67 (red color) and angiogenesis markers CD31 (green color) and $\alpha$-SMA (red color) and detected using confocal microscopy (PerkinElmer UltraVIEW Vox, magnification, $\times 200$ or $\times 400$, nine continual fields put together). Right, the tissue sections were screened under a low-power field, and five fields were selected. Each field was analyzed separately to obtain the fraction of apoptotic cells, ki67 positive cells fluorescent areal density and microvessel density. The data is presented as mean \pm SD across the vision fields $(*, p<0.05 ; * *, p<0.01 ; * * *, p<0.001)$. SD, standard deviation; TUNEL, terminal deoxynucleotidyl transferase deoxyuridinetriphosphate nick end labelling; SMA, smooth muscle actin.

The BM microenvironment of MM hosts and related cytokine secretion had been reported to be associated with proliferation, survival, migration and drug resistance in MM cells [33]. In this manuscript, two different SCID-rab mouse models were used to investigate the potency of NNU546 against MM in the presence of the $\mathrm{BM}$ microenvironment, which were similar to the SCID-hu model [34]. In this SCID-rab model, RPMI 8226-Luc cells expressing a luciferase reporter gene were directly injected into rabbit bone chips that were implanted subcutaneously in SCID mice and $\mathrm{MM}$ tumor growth was tracked by quantitative Xenogen imaging in living animals. A robust anti-tumor response was observed in mice receiving an oral dose of $1 \mathrm{mg} / \mathrm{kg}$ of NNU546 once daily compared with vehicle (Figure 6A, 6B; $p<0.01$ ). The H\&E staining of histological sections of the implanted bone of MMbearing SCID-rab mice revealed massive myeloma cell infiltration and increased osteoclast activity in control group (Figure 6C). In contrast, implanted bone of a host treated with NNU546 showed no obvious myeloma cells survival, while an increased number of trabecular bone and osteoblasts were observed.

We next assessed the level of circulating human monoclonal $\mathrm{IgG}$ in mouse serum as a marker for monitoring the tumor burden in SCID-rab models. The therapeutic efficacy of drugs against MM-induced bone disease was evaluated by measuring the implanted bone mineral density (BMD) changes by the microCT system. The association between the anti-myeloma and bone anabolic effect of NNU546 was visualized by Xradiographs. As shown in Figure 6D, osteolytic bone lesions were evident in implanted bones of control hosts at the end of the experiment. MicroCT imaging of bone grafts revealed that great portions of the bone graft in the control group were eroded (Figure 6E) and that trabecular bone was reduced (Figure 6F). Tumor burden assessed by the human monoclonal $\mathrm{IgG}$ content in serum showed that the decrease of IgG levels in the NNU546 group paralleled the significant reduction of tumor volume compared with the vehicle group (Figure $6 \mathrm{G} ; \mathrm{p}<0.01)$. Moreover, in contrast to control animals, the structure of the bone grafts in mice that had received NNU546 therapy was well preserved. In addition, the BMD of the implanted bone treated with NNU546 was higher than that of the control (Supplementary Figure 4A). The histomorphometric analysis revealed that the bone volume/total volume, trabecular thickness and trabecular number in myelomatous bones treated with NNU546 were all increased (Supplementary Figure 4B4D), indicating that NNU546 reduced bone loss in the grafts. Histological examination of bone grafts treated with NNU546 revealed that the osteoblasts were increased and the multinucleated osteoclasts were reduced compared with those in the vehicle group (Figure 6H).

In summary, the results from 3 different MM xenograft mouse models demonstrated the potent in vivo antitumor activity of NNU219/NNU546 at welltolerated doses. The results obtained with the SCID-rab model provided in vivo evidence for the ability of NNU219/NNU546 to trigger apoptosis of tumor cells even in the presence of the BM microenvironment.

\section{DISCUSSION}

Proteasome inhibition is a highly effective mechanism of action in MM treatment. The first-generation proteasome inhibitor bortezomib was approved for the treatment of $\mathrm{MM}$ and provided a great benefit for patients. However, despite significant improvements in the survival of MM patients, frequent administration led to development of drug resistance and toxic effects, particularly peripheral neurotoxicity (PN). Carfilzomib, the second-generation proteasome inhibitor, was associated with a low incidence of PN, but serious adverse events (SAEs) and was intravenously administered [23, 35]. As most patients become resistant against these drugs or can no longer tolerate treatment due to specific side effects such as neurotoxicity (bortezomib) or cardiotoxicity (carfilzomib), there is an urgent need to develop a novel well tolerated orally applicable proteasome inhibitor effective in bortezomib and/or carfilzomib resistant patients. In this study, a novel orally administered 
A

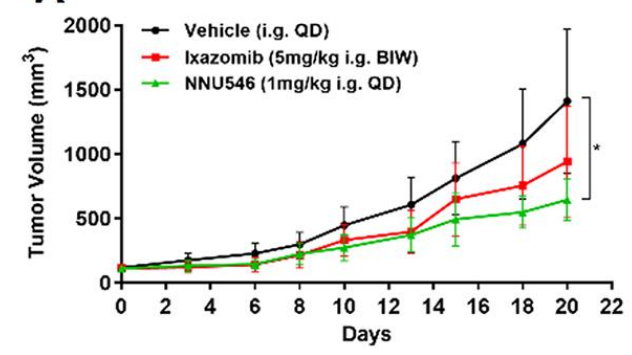

B

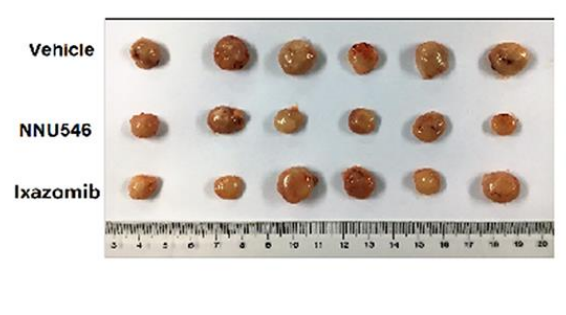

C

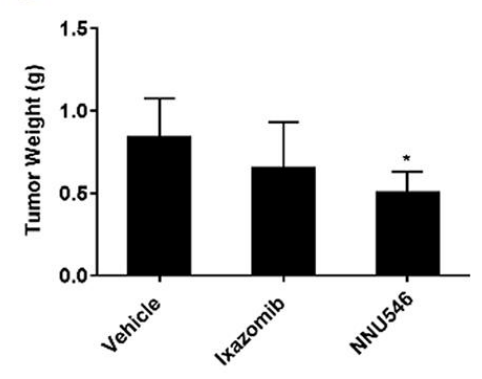

D
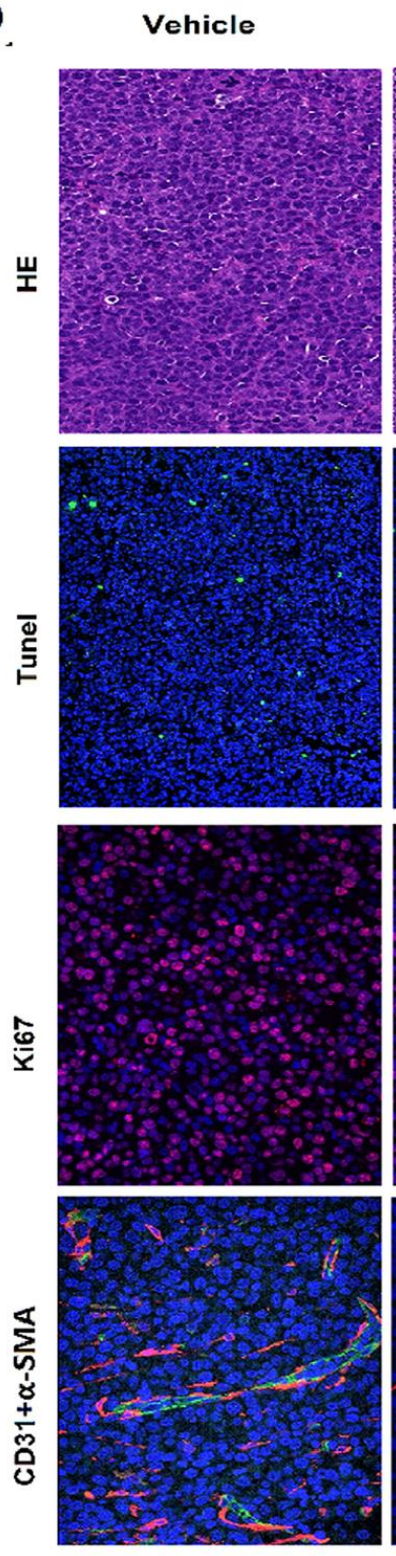

Ixazomib
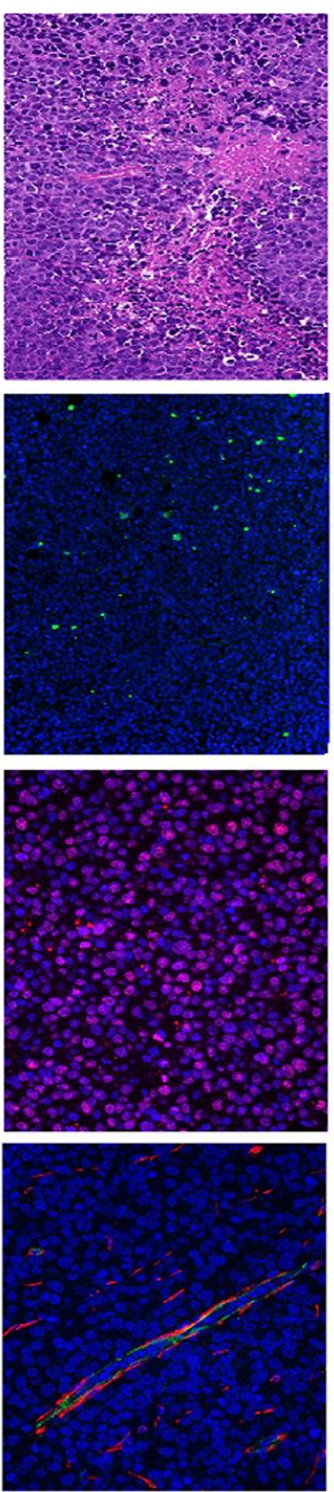

NNU546
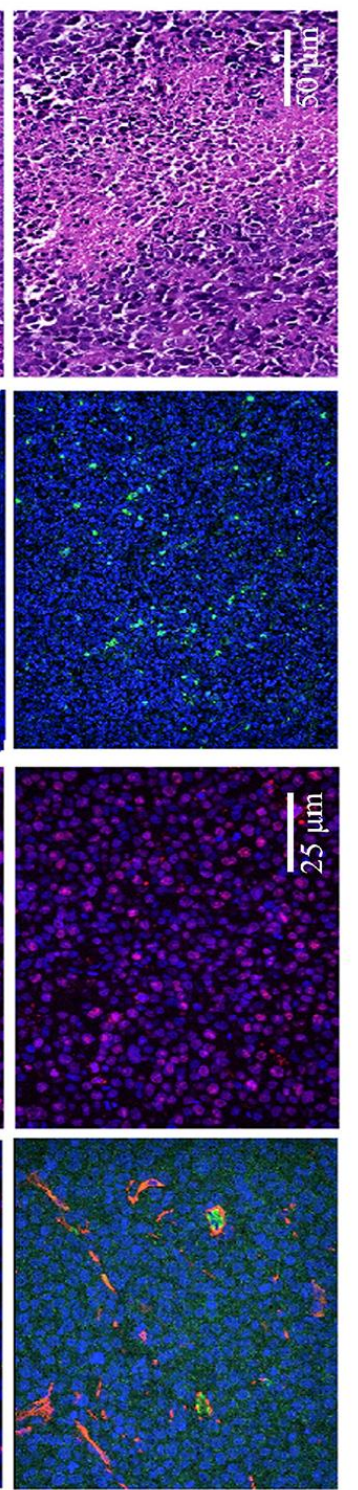
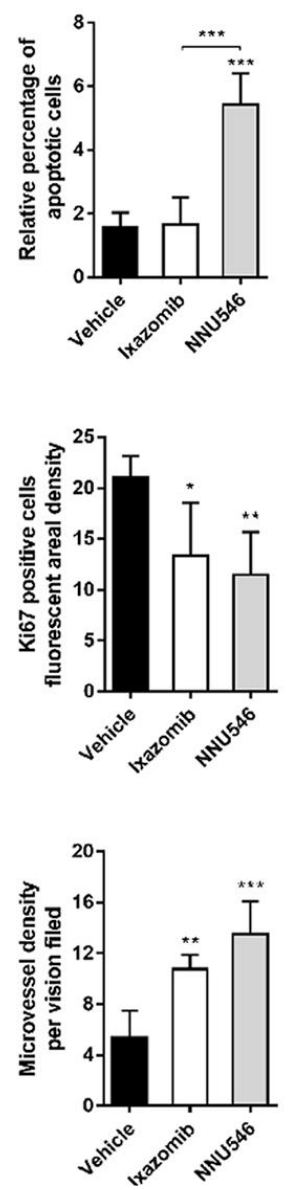

Figure 5. Effects of NNU546 and ixazomib on tumor growth in PDX model. (A) Relative tumor growth of PDX models treated orally with vehicle, ixazomib (5 mg/kg, BIW) or NNU546 (1 mg/kg, QD) scheduled for 3 weeks. Data were presented as mean \pm SD $\left(n=6 ;{ }^{*}, p<0.05\right)$. (B) Differences in tumor size for the vehicle and treatment groups. The tumors were resected from the NCG mice at the end of the experiment. (C) Average tumor weight in the vehicle and treatment groups. Data were shown as mean \pm SD $(*, p<0.05)$. (D) Left, H\&E and immunostaining of tumor sections of vehicle, ixazomib and NNU546 treated mice, including apoptosis-associated TUNEL (green color), 
proliferation marker Ki-67 (red color) and angiogenesis markers CD31 (green color) and $\alpha$-SMA (red color), detected using confocal microscopy (PerkinElmer UltraVIEW Vox, magnification, $\times 200$ or $\times 400$, nine continual fields put together). Right, the tissue sections were screened under a low-power field, and five fields were selected. Each field was analyzed separately to obtain the fraction of apoptotic cells, ki67 positive cells fluorescent areal density and microvessel density. The data is presented as mean \pm SD per across the fields, $(*, p<0.05 ; * *$, $p<0.01 ; * *, p<0.001$ ). TUNEL, terminal deoxynucleotidyl transferase deoxyuridinetriphosphate nick end labelling; PDX, patient-derived xenograft; SMA, smooth muscle actin; SD, standard deviation.

bioactive dipeptidyl boronic acid candidate, NNU546, was demonstrated to have potent in vitro anti-cancer activity and in vivo efficacy in different xenograft mouse models of MM.

The enzymatic activities of NNU219 (a biologically active form of NNU546 to which it hydrolyzes upon exposure to aqueous solutions or plasma) against the proteasome were evaluated. NNU219 significantly inhibited the CT-L subunits of constitutive proteasome and immunoproteasome in a dose-dependent manner with minimal inhibition of T-L and C-L activities. Theoretical docking results revealed the binding mode of NNU219 with $\beta 5$ subunit (five strong hydrogen bonds were formed except an additional hydrophobic interaction). This indicates that NNU219 was able to effectively bind proteasome. Recently, it was demonstrated that hematologically derived cells expressed the subunits $\beta 5 \mathrm{i}$ and $\beta 5 \mathrm{c}$, and inhibition of only one subunit did not alter cell viabilities [36]. Inhibition of CT-L activity represents a valid and successful anti-tumor strategy in hematological tumors without causing cytotoxicity in non-transformed cells [37]. Similar results on the selectivity among the three subunits were obtained in different MM cell lines and mice. It revealed that NNU219 inhibited all three proteasome activities in MM cell lines, which was different from the results of the enzymatic assay. The differences between the proteasome and cells were possibly due to the specific environment in the cell, including metabolism of compounds, protein-protein interactions, post-translational modifications and asymmetric intracellular localization of compounds [38]. Furthermore, the activity of proteasome in cell slowly recovered after drug exposure, which was possibly caused by the induction of mRNA transcription and de novo synthesis of individual subunits and their further transformation into a new proteasome [30].

In the present study, it was demonstrated for the first time that NNU219 markedly inhibited cell growth in various MM cell lines, including those that were sensitive and resistant to conventional therapies with various cytogenetic backgrounds. Although the activity of NNU219 varied among different MM cell lines, it was more active against MM.1S and MM.1R cells with wild-type p53. P53 and its downstream genes encoding the proteins p21 and p27 are endogenous cyclindependent protein kinase inhibitors, and suppressed cell cycle progression [22]. Our study indicated that NNU219 induced the expression of p53, p21 and p27 in MM cells. Treatment with NNU219 also caused upregulation of the expressions of cyclin B1 and CDK1. As the degradation of these proteins is mediated by the ubiquitin-proteasome pathway, it was speculated that NNU219 might block proteasome activity, resulting in inhibition of the degradation of cyclin B1 and CDK1, which leads to their accumulation. As it was not possible to normally activate CDK1, cells were not able to enter mitosis and were arrested in $\mathrm{G} 2 / \mathrm{M}$ phase of the cell cycle, resulting in induction of apoptosis. The flowcytometry results further confirmed that inhibition of cell growth was attributed to induction of apoptosis. NNU219 induced apoptosis by activation of the caspase cascades, which was reflected by increased cleavage of caspase-9, caspase-8, caspase-3 and PARP, suggesting that NNU219 triggered the intrinsic and extrinsic pathways of apoptosis. Furthermore, NNU219 caused accumulation of proteasome substrates, including polyubiquitinated proteins, which then led to activation of UPR, as evidenced by the activation of ATF4 and $\mathrm{XBP} 1$, and the induction of BIP and CHOP expression. Of note, the present results suggested that NNU219 reduced the viability of tumor cells of patients with primary MM who exhibited disease progression after various treatments without affecting the viability of normal PBMCs. Results obtained from multiple cell lines showed that proliferating malignant cells were affected by NNU219 due to the accumulation of damaged proteins at a much higher rate than that in normal quiescent cells [39, 40].

NF- $\mathrm{kB}$ is a key regulator of growth and survival of MM cells. It is chronically active in MM cells and may be induced by cytokines, prosurvival factors, chemokines and contact with the components of microenvironment. Many studies have demonstrated that bortezomib prevented I $\mathrm{I} B \alpha$ from degradation by proteasome, thus inhibiting nuclear translocation of $\mathrm{NF}-\kappa \mathrm{B}$, cytokine circuits and the survival advantage for MM cells conferred by the BM microenvironment [6, 34, 41]. Previous studies suggested that the anti-cancer activity of bortezomib in $\mathrm{MM}$ might be attributed to the inhibition of inducible NF- $\kappa \mathrm{B}$ activity $[6,32]$. However, recent evidence suggested a paradigm shift in this mechanism [42]. Bortezomib enhanced constitutive NF$\kappa \mathrm{B}$ activity via activation of the canonical pathway, which suggested that bortezomib-induced cytotoxicity 
A

Pre-Rx

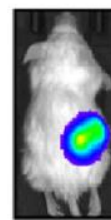

Final

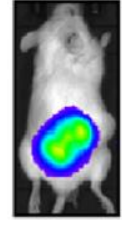

Vehicle

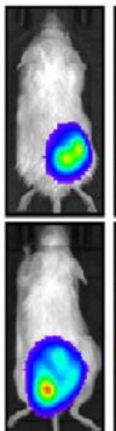

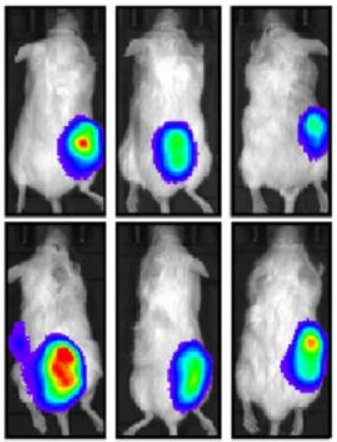

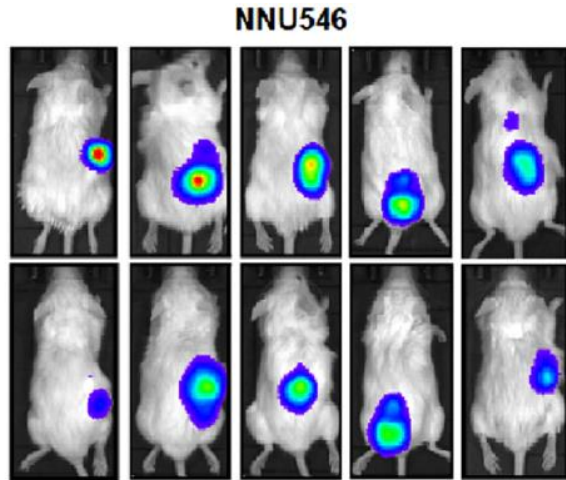

B

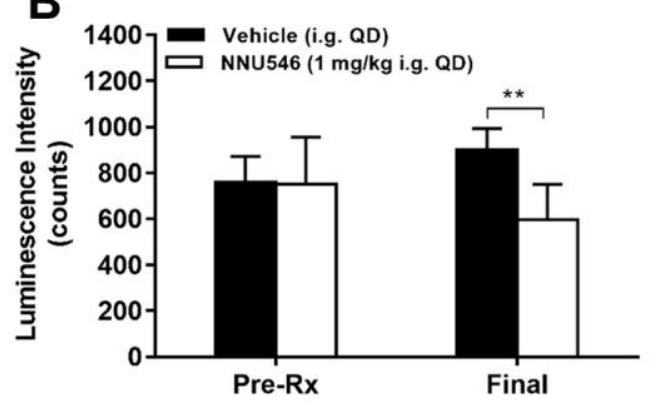

C

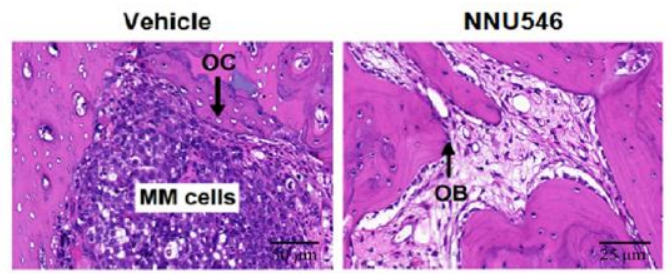

G

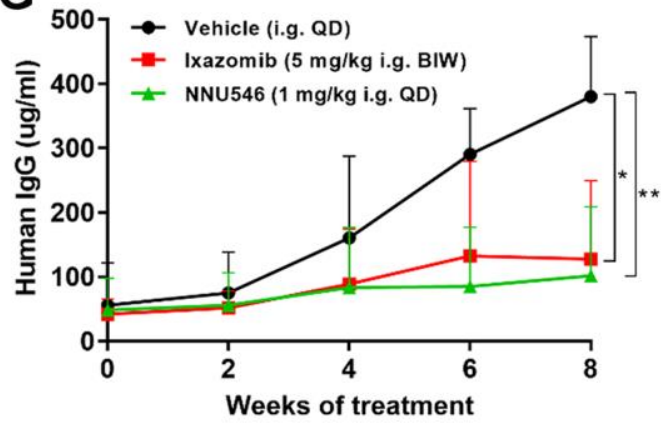

D

E
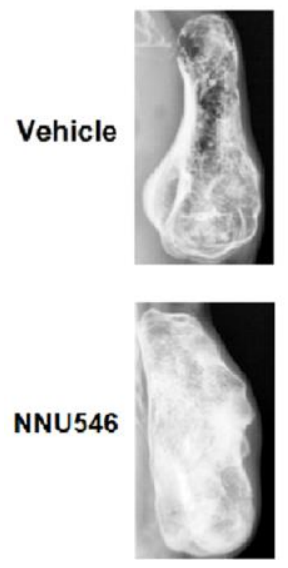

F
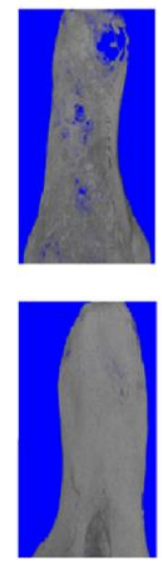
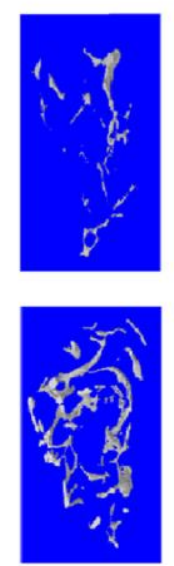

H

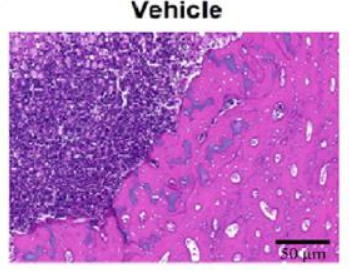

NNU546

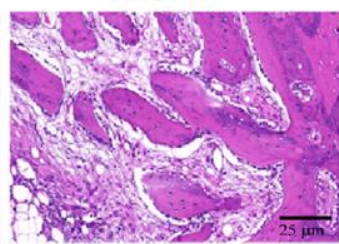

Figure 6. NNU546 inhibits the growth of human MM cells in SCID-rab mouse model. (A) Rabbit bone grafts were subcutaneously implanted into SCID mice. After four weeks, $5 \times 10^{6}$ of RPMI 8226 cells with luciferase expression were injected directly into the rabbit bone in SCID mice. After establishment of this in vivo model, mice were orally administered with either vehicle or NNU546 (1 mg/kg, QD). Representative imaging of the live animals demonstrated the luciferase expression prior to treatment (Pre-Rx) and at the end of the experiment (Final) in mice. (B) Luminescence intensity was quantified using living animal imaging of mice prior to treatment (Pre-Rx) and at the end of the experiment (Final). Data were expressed as mean $\pm S D(n=5 ; * *, p<0.01)$. (C) H\&E staining of histological sections of myelomatous bones engrafted with myeloma cells ( $\times 200$ or $\times 400$ original magnification). Increased myeloma cell infiltration and osteoclast activity were noted in control group. In contrast, myelomatous bone from a host treated with NNU546 had no apparent myeloma cells but possessed increased number of trabecular bone and osteoblast. (D) SCID-rab mice engrafted with myeloma cells from MM patients were treated with vehicle, ixazomib (5 mg/kg, BIW) or NNU546 (1 mg/kg, QD). X-radiographs of myelomatous bones engrafted with myeloma cells from MM patients were performed at the end of the experiment. (E, F) Representative microcT images of bone grafts excised from vehicletreated animals and mice that had received NNU546 for 8 weeks. (G) Effect of therapy on myeloma growth assessed by human IgG levels in the mouse serum measured by ELISA. Data were shown as mean $\pm \mathrm{SD}\left(\mathrm{n}=5 ;{ }^{*}, p<0.05 ; * *, p<0.01\right)$. (H) H\&E staining of histological sections of myelomatous bones engrafted with cells from MM patients (original magnification, $\times 200$ or $\times 400$ original magnification). 
might not be completely ascribed to the inhibition of canonical NF- $\mathrm{KB}$ activity in MM [23, 31]. In vitro studies indicated that NNU219 inhibited the NF- $\mathrm{kB}$ activation through promoting the accumulation of ubiquitination. In addition, NNU219 caused downregulation of the expression of several downstream effectors of NF- $\kappa \mathrm{B}$. The expression of these NF- $\kappa \mathrm{B}$-mediated genes is known to affect growth factors, cell adhesion, angiogenesis and resistance to chemotherapeutic agents and radiotherapy [6].

By testing in vivo pharmacokinetic and pharmacodynamic characteristics, NNU546 was identified as an orally bioavailable candidate. The biodistribution and inhibition profile of proteasome activities of NNU546 in vivo was investigated. Oral administration of NNU546 exerted significant proteasome inhibition in whole blood at $1 \mathrm{~h}$, which was recoverable at $168 \mathrm{~h}$, whereas bortezomib inhibited proteasome activity with only minimal or no inhibition at $48 \mathrm{~h}$. In all tissues except the brain, recovery of the proteasome activity from NNU219/NNU546-mediated inhibition was comparable to that of bortezomib and the recovery of proteasome activity in the tissues was faster than in the blood, indicating that new proteasome synthesis had a dominant role in the recovery of proteasome activity in tissues other than in whole blood. This might be due to the inability of mature erythrocytes to restore their activity by generating new proteasomes, whereas the recovery of proteasome activity in whole blood depended on the production of new erythrocytes.

To confirm the in vitro results, the present study also employed several types of xenograft mouse models derived from human MM to investigate the efficacy of the compounds in vivo. The results indicated that NNU219/NNU546 significantly inhibited tumor growth and prolonged the survival of mice in all MM animal models. The results obtained using the MM xenograft models indicated that the significant and sustained antitumor efficacies achieved by intravenous or oral administration of NNU219/NNU546 were associated with the pharmacodynamics of the drugs. Although xenografts derived from human tumor cell lines allowed for rapid evaluation and prioritization of potential drug candidates for progression to clinical trials, these models did not fully reflect the biology and heterogeneity of their human disease counterparts. To reduce intra-tumoral heterogeneity, PDX models were established in mice to more accurately model the characteristics of human tumors. The results indicated that treatment with NNU546 in PDX models significantly inhibited the tumor growth compared with the control group. Moreover, no significant toxicity was observed in NNU546 treatment group. The potent antiMM activity of NNU546 in vivo was confirmed by IF analysis of tumor sections of control and NNU546 treated mice. The results suggested that NNU546 induced MM cell apoptosis (TUNEL) and decreased the expression of MM cell proliferation (Ki-67) and angiogenesis (CD31 and $\alpha$-SMA). The SCID-rab mouse model reflected the interactions between the human BM microenvironment and human MM cells. Importantly, our data provided evidence that NNU546 directly targeted MM cells and overcame the cytoprotective effects of the BM microenvironment on MM cells.

In conclusion, our results demonstrated that compound NNU546 was a novel orally active candidate and being investigated as a new drug. We believe that this new candidate compound will benefit for the MM patients for better curative effect and less side effects.

\section{MATERIALS AND METHODS}

\section{Cell cultures}

The human myeloma cell lines MM.1S, MM.1R, U266, RPMI 8226, ARH77, HCC1937, MDA-MB-231, A549, SCG7901 and BxPC-3 (all from the American Type Culture Collection, USA) were cultured in RPMI1640 medium supplemented with $10 \%$ fetal bovine serum (FBS) and 1\% sodium pyruvate. All cell lines were routinely maintained at $37{ }^{\circ} \mathrm{C}$ in $5 \% \mathrm{CO}_{2}$. After FicollHypaque density gradient centrifugation, mononuclear cells from patients' $\mathrm{BM}$ aspirates were subjected to CD138 (Syndecan-1) purification using Micro Beads and the Auto MACS magnetic cell sorter (Miltenyi Biotech, Bergisch Gladbach, Germany) according to the manufacturer's protocol. CD138-positive cells with a purity of $>95 \%$ verified by flow cytometric analysis were used for further experiments. Peripheral blood mononuclear cells (PBMCs) were obtained from healthy individuals after Ficoll-Hypaque density separation as previously described [33]. Informed consent was obtained from all patients and volunteers in accordance with the Declaration of Helsinki. The ethical number is 2013-SRFA-067.

\section{Enzyme activities and inhibition assays}

Inhibition of CT-L, C-L, and trypsin-like (T-L) activities of $20 \mathrm{~S}$ proteasome was assessed using purified human erythrocyte-derived constitutive $20 \mathrm{~S}$ proteasomes (cCP) and 20S immunoproteasome (iCP; Boston Biochem, Cambridge, MA, USA), as previously described [43, 44]. Standard substrate-based assays were initiated by enzyme in the presence of $10 \mu \mathrm{M}$ of the test compounds for the non-proteasome protease panel assay (GenScript, Nanjing, China) as previously described [29]. 


\section{Docking studies}

To investigate the binding mode of NNU219 with $\beta 5$ subunit of proteasome, in silico modelling of docking was carried out. The covalent docking was performed using the Glide module of Schröedinger software (LLC, New York, NY, USA, 2015). The THR1 residue of $\beta 5$ subunit of proteasome (PDB ID: 4R67) was covalently anchored with the boronic acid fragments of NNU219 and MLN2238, respectively. And other groups of the molecules were docked with the residues of the proteasome.

\section{Intracellular and in vivo proteasome activity assays}

For the cellular proteasome activity assays, cells were treated with concentrations of compounds $(0-10 \mu \mathrm{M})$ for $1 \mathrm{~h}$ at $37{ }^{\circ} \mathrm{C}$ and the CT-L, C-L and T-L activities of proteasome were evaluated by using the Proteasome-Glo assay reagents (Promega Corp. Madison, WI, USA) according to the manufacturer's protocol. For determination of recovery of cellular proteasome activity, cells were treated with the compounds for $1 \mathrm{~h}$ and then washed thrice in PBS to remove the compounds. Cells were incubated in fresh medium for an additional 4, 24 or $72 \mathrm{~h}$ at $37^{\circ} \mathrm{C}$. To investigate the in vivo proteasome activities, female beige nude xid (BNX) mice (5-7 weeks old; Shanghai Sippr-BK laboratory Animal Co. Ltd.) were intravenously administered with a single dose of NNU219 $(0.2$ or $0.4 \mathrm{mg} / \mathrm{kg})$ or bortezomib $(1 \mathrm{mg} / \mathrm{kg})$, respectively. Blood samples were collected after $1 \mathrm{~h}$, and whole blood cells were then analyzed for proteasome activity as previously described [45].

\section{Cell viability, cell cycle and apoptosis assays}

Cell viability assay was determined using a Cell Counting Kit-8 (CCK-8; Dojindo Laboratories, Kumamoto, Japan). In brief, cancer cells were suspended and seeded in the culture medium in a 96well plate. After $24 \mathrm{~h}$, cells were treated with the compounds at $37{ }^{\circ} \mathrm{C}$ for $1 \mathrm{~h}$ or $72 \mathrm{~h}$ followed by washing thrice with PBS and further incubation in fresh medium for an additional $72 \mathrm{~h}$. The CCK8 reagent was added to each well and the plates were incubated at 37 ${ }^{\circ} \mathrm{C}$ for another 1-2 $\mathrm{h}$. The absorbance was measured with a microplate reader (CLARIOstar; BMG Labtech) at $450 \mathrm{~nm}$. Cell viability was also assessed by exclusion of trypan-blue staining. The viabilities of CD138positive cells and PBMCs were assessed using the Cell Titer-Glo Luminescent Cell Viability Assay kit (Promega, Corp., Madison, WI, USA) with cells plated and processed as described above. Cellular DNA content was quantified using a propidium iodide (PI) staining assay kit (BD Biosciences, San Diego, CA, USA). The percentage of apoptotic cells was detected using the Annexin V/PI staining assay kit (BD Biosciences, San Diego, CA, USA). Cell cycle and apoptosis were analyzed by flow cytometry (FACSCalibur, BD Biosciences).

\section{Western blot analysis}

Western blot analysis was performed as previously described [46]. In brief, equal amounts of protein were prepared, resolved on 15\% SDS-PAGE and transferred onto PVDF membranes. Membranes were blocked by incubation in 5\% nonfat dry milk in Tris-buffered saline containing Tween-20 (TBST) for $1 \mathrm{~h}$, and probed with specific antibodies to ubiquitinated proteins (\#3933, Cell Signaling Technology, MA, USA), caspase-3 (\#9662, Cell Signaling Technology, MA, USA), caspase-8 (\#4927, Cell Signaling Technology, MA, USA), caspase-9 (\#9502, Cell Signaling Technology, MA, USA), BIP (\#3183, Cell Signaling Technology, MA, USA), phosphorylated (p)-eukaryotic translation initiator factor (EIF) $2 \alpha$ (\#9721, Cell Signaling Technology, MA, USA), X-box binding protein (XBP)1 (\#83418, Cell Signaling Technology, MA, USA), activating transcription factor (ATF)4 (\#11815, Cell Signaling Technology, MA, USA), CHOP (\#2895, Cell Signaling

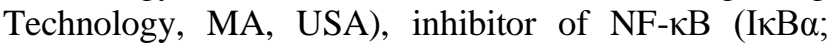
\#9242, Cell Signaling Technology, MA, USA), p-IкB $\alpha$ (Ser32; \#2859, Cell Signaling Technology, MA, USA), p65 (\#6956, Cell Signaling Technology, MA, USA), $\beta$ actin (\#4970, Cell Signaling Technology, MA, USA), poly (ADP ribose) polymerase (PARP; \#551024, BD Bioscience Pharmingen, San Diego, CA, USA), p53 (sc47698, Santa Cruz Biotechnology, Inc., CA, USA), p21 (sc-24559, Santa Cruz Biotechnology, Inc., CA, USA), Cyclin B1 (sc-70898, Santa Cruz Biotechnology, Inc., CA, USA), cyclin-dependent kinase (CDK1; sc-53219, Santa Cruz Biotechnology, Inc., CA, USA), PERK (sc377400, Santa Cruz Biotechnology, Inc., CA, USA) and GAPDH (sc-69778, Santa Cruz Biotechnology, Inc., CA, USA) at $4{ }^{\circ} \mathrm{C}$ for $12 \mathrm{~h}$, respectively. After washing with TBST, membranes were incubated with a peroxidase-conjugated secondary antibody (Bioworld, Nanjing, China) at room temperature for $1 \mathrm{~h}$. Blots were visualized using enhanced chemiluminescence reagents (Bioworld, Nanjing, China). The experiments are performed for three times.

\section{Co-immunoprecipitation (Co-IP) and ubiquitination}

Co-IP was performed as previously described [47]. In brief, the MM cell lines MM.1S and RPMI 8226 were treated with $10 \mathrm{nM}$ of NNU219 for $24 \mathrm{~h}$. The control group was incubated in the same concentration of DMSO in culture medium. The stimulated cells were lysed in western and IP buffer (Beyotime, Jiangsu, China) and centrifuged at $4^{\circ} \mathrm{C}$. Correlative proteins were 
immunoprecipitated from $1 \mathrm{mg}$ MM cell lysate using $\operatorname{I\kappa B} \alpha(1: 1000)$ or $\operatorname{IgG}(1: 1000)$ antibody (rabbit) and coupled to protein $\mathrm{A} / \mathrm{G}$ agarose beads (Beyotime, Jiangsu, China) overnight at $4{ }^{\circ} \mathrm{C}$. The beads were washed with IP buffer, and the elution was processed by ubiquitin or I $\mathrm{I} \mathrm{B} \alpha$ immunoblotting.

\section{NF-кB inhibition assay}

To determine the inhibition of NF- $\mathrm{KB}$ signaling, the transfected NF- $\kappa \mathrm{B} /$ luciferase $293 \mathrm{~T}$ cells were pretreated with various concentrations of NNU219 or bortezomib for $6 \mathrm{~h}$ and then stimulated with $10 \mathrm{ng} / \mathrm{ml}$ of tumor necrosis factor- $\alpha$ (TNF- $\alpha$ ) for $18 \mathrm{~h}$, followed by measurement of luciferase activity by using the DualLuciferase $^{\circledR}$ Reporter Assay System (Promega Corp., Madison, WI, USA) as previously described [46].

\section{Real-time quantitative reverse transcriptase- polymerase chain reaction (RQ-PCR)}

Total RNA was extracted using TRIzol reagent (Invitrogen; Thermo Fisher Scientific, Inc., USA). Realtime qPCR was performed using the RevertAid First Strand cDNA Synthesis Kit (Thermo Fisher Scientific, Inc., Waltham, MA, USA) and FastStart Universal SYBR Green Master (Rox; Roche) according to the manufacturer's protocols. PCR reaction was performed in a $25-\mathrm{mL}$ volume, and the thermocycling conditions were $95{ }^{\circ} \mathrm{C}$ for $30 \mathrm{~min}$ followed by 40 cycles of $95{ }^{\circ} \mathrm{C}$ for $60 \mathrm{sec}$ and $60{ }^{\circ} \mathrm{C}$ for $20 \mathrm{sec}$. The following are the primer sequences: H-ICAM1-S, 5'-CCGTTGCCTAAA AAGGAGTTGC-3'; H-ICAM1-A, 5'-TGGCAGCGTA GGGTAAGGTTC-3';5'-H-TNF- $\alpha$-STCTACTCCCAGG TCCTCTTCAAG- 3 '; 5 '-H-TNF- $\alpha$-AGGAAGACCCCT CCCAGATAGA-3';5'-H-IL-6-S, GTAGTGAGGAACA AGCCAGAGC-3'; 5'-H-IL-6-A, TACATTTGCCGAA GAGCCCT-3'; 5'-H-VEGF-S, GGAGGGCAGAATCA TCACGA-3'; 5'-H-VEGF-AGCTCATCTCTCCTATGT GCTGG-3'. All PCRs were run in triplicate, and the mRNA levels of target genes relative to $\beta$-actin were calculated using the $2^{-\Delta \Delta \mathrm{CT}}$ method [48].

\section{Microsomal stability assay}

Microsomal stabilities were determined by incubating 1 $\mu \mathrm{M}$ of the test compounds with rat, mouse, dog, monkey or human liver microsomes (Xenotech, USA) for different durations at $37{ }^{\circ} \mathrm{C}$. Clearance and $\mathrm{T}_{1 / 2}$ were calculated by measuring the remaining percentage of test compounds at different time-points. Liquid chromatography/mass spectrometry (Xevo Quadrupole Timeof-Flight Mass Spectrometer; Waters) and an ACQUITY High-Performance Liquid Chromatography (HPLC) system (Shimadzu, Japan) were used to determine the relative peak area for the parent compounds.

\section{Pharmacodynamic study}

For the pharmacodynamic study, female BNX mice ( $\mathrm{n}=6$; 5-7 weeks old; Shanghai Sippr-BK laboratory Animal Co. Ltd.) were given one single dose of NNU219 $(0.4 \mathrm{mg} / \mathrm{kg})$ and bortezomib $(1 \mathrm{mg} / \mathrm{kg})$ by i.v. administrations or NNU546 $(2-15 \mathrm{mg} / \mathrm{kg})$ by oral gavage. At different time-points after drug administrations, $100 \mu \mathrm{L}$ of whole blood was collected from each mouse and processed for the $20 \mathrm{~S}$ proteasome activity assay. After 1 or $24 \mathrm{~h}$ post-drug administration, tissue samples (heart, liver, spleen, lung, brain and tumors) were collected and homogenized in PBS buffer with three volumes of lysis buffer. Tissue homogenates and cell lysates were removed by centrifugation and supernatants were collected for protein quantitation and proteasome activity determination using the Proteasome-Glo assay reagents as described above [45].

\section{In vivo activity of NNU219 and NNU546 in ARH77 MM xenograft models}

All experiments were performed according to protocols approved by the Institutional Animal Care and Use Committee of Nanjing Normal University. All animals were housed for 1-2 weeks prior to the experiment and maintained in a controlled environment with free access to food and water ad libitum.

For the ARH77 xenograft model, female BNX mice $(\mathrm{n}=5 ; 5-7$ weeks old; Shanghai Sippr-BK laboratory Animal Co. Ltd.) were implanted with $5 \times 10^{6}$ of ARH77 cells in Matrigel (1:1) and randomized into treatment and vehicle groups when the mean tumor volume (MTV) reached $100-150 \mathrm{~mm}^{3}$. For the intravenous treatment groups, mice were treated with bortezomib (1 $\mathrm{mg} / \mathrm{kg}, \quad \mathrm{BIW})$ or NNU219 (0.4 mg/kg, BIW), respectively. For i.g. administration, mice were treated with ixazomib (5 mg/kg, BIW) and NNU546 (1 mg/kg, QD) or NNU546 (2 mg/kg, QOD). Tumors were measured twice a week and the tumor volume was calculated using the following formula: $0.5 \times$ (length $\times$ width $^{2}$ ). Animals were euthanized when the tumors reached $2 \mathrm{~cm}^{3}$ or exhibited visible signs of necrosis.

\section{In vivo activity of NNU546 in PDX model}

The PDX model was generated from biopsies of patients with MM brain manifestation at the Department of Hematology, the 1st Affiliated Hospital of Nanjing Medical University. Freshly excised tumor tissue specimens were washed and cut into $2.5 \times 2.5 \times 2.5 \mathrm{~mm}^{3}$ pieces in antibiotic-containing L-15 medium. Under anesthesia with pentobarbital, 4-6 weeks-old male NCG mice ( $n=6$; Model Animal Research Center of Nanjing University) were subcutaneously transplanted with the 
tumor pieces. Tumors were harvested when their size reached $500 \mathrm{~mm}^{3}$ (Px1 xenografts). Tumor tissue from Px1 xenograft mice was divided into small pieces and then implanted again subcutaneously as described above to obtain Px2 xenografts. This process was once more and the experiments were performed on Px3 xenograft mice, which were randomized into treatment groups when tumor volume reached $100-150 \mathrm{~mm}^{3}$. Mice were orally administered with ixazomib $(5 \mathrm{mg} / \mathrm{kg}$, i.g., BIW) or NNU546 (1 mg/kg, QD). Tumors were measured twice a week.

\section{In vivo activity of NNU546 in a SCID-rab model}

For the SCID-rab model, RPMI 8226 cells were transfected with luciferase-expressing lentivirus and designated as RPMI 8226-Luc, which were then injected into rabbit bone fragments implanted subcutaneously into SCID mice (Shanghai Sippr-BK Laboratory Animal Co. Ltd.) as previously described [49]. The tumor burden was determined by monitoring the fluorescence activity with an IVIS 200 imaging system (Xenogen) [34]. Mice were orally administered with ixazomib (5 $\mathrm{mg} / \mathrm{kg}, \mathrm{BIW}$ ) or NNU546 (1 mg/kg, QD).

For the construction of the primary myelomatous SCIDrab mouse model, unseparated myeloma BM cells from patients were injected directly into rabbit bone implanted subcutaneously into SCID mice (Shanghai Sippr-BK Laboratory Animal Co. Ltd.). The oral administrations of ixazomib and NNU546 were identical to those in the RPMI 8226-Luc SCID-rab model. Tumor growth was determined by the IgG levels in the blood of the mice using enzyme-linked immunosorbent assay (ELISA; FCMZCS, Nanjing, China) [49]. At the end of the experiment, bone grafts were radiated in 5-Gy fractions (total dose, 40 Gy) using a Faxitron CP-160 X-ray generating system (Faxitron X-Ray Corp., Wheeling, IL) at a dose rate of $0.5-1.5 \mathrm{~Gy} / \mathrm{min}$ with $100 \mathrm{kVp}$ and $10 \mathrm{~mA}$. The grafts were gently extended into the radiation field with the remainder of the body of the anesthetized mouse shielded by custom-made cerrobend blocks [39]. Changes in bone mineral density (BMD) of the implanted bone and the femur of the mice were assessed using a microcomputed tomography (microCT) system ( $\mu \mathrm{CT} 40$; SKYSCAN). The bone grafts were scanned for qualitative assessment of the microarchitectureon $\mu \mathrm{CT} 40$ using the manufacturer's software (CTan 1.10, Skyscan). Horizontally sectioned images were obtained with a voxel size of $9 \mu \mathrm{m}$. Semi-automated contouring was used to select a region of interest extending through the whole length of the grafts, composed of 100 adjacent individual two-dimensional slices. Analysis of the trabecular microarchitecture in the distal femur was performed using CTan program 1.10 [40].

\section{Histology and immunohistochemistry (IHC)}

At the end of the in vivo activity experiments, mice were sacrificed, and tumors were immediately fixed in $10 \%$ of formalin for $24 \mathrm{~h}$, and were then washed, dehydrated and embedded in paraffin. The bones were fixed, decalcified and embedded in paraffin. Finally, staining with hematoxylin-eosin (H\&E) was performed using tumor or bone sections which were mounted on poly-lysine slides. On the hand, the main process of IHC is as follows: endogenous peroxidase activity was blocked with $3 \%(\mathrm{v} / \mathrm{v}) \mathrm{H}_{2} \mathrm{O}_{2}$ for $15 \mathrm{~min}$. After blocking with $5 \% \mathrm{BSA}$ at $37^{\circ} \mathrm{C}$ for $0.5 \mathrm{~h}$, the antigen was thermally repaired with citrate. The purified 14-3-3 $\tau$ monoclonal antibody (Proteintech Group, Chicago, IL, USA) was incubated overnight at $4^{\circ} \mathrm{C}$. Afterwards, using secondary antibody for $0.75 \mathrm{~h}$ at $37^{\circ} \mathrm{C}$, followed by dropping $\mathrm{SABC}$ at $37^{\circ} \mathrm{C}$ for $30 \mathrm{~min}, \mathrm{DAB}$ coloring, and finally counterstaining with hematoxylin. The histologic and IHC images were obtained by light microscopy using an optical microscope Nikon i55 (Nikon Corp., Tokyo, Japan) and analyzed.

\section{Immunofluorescent (IF) analysis of apoptosis and angiogenesis}

The tumor sections obtained from xenograft mice described above were subjected to IF staining for Ki-67 or terminal deoxynucleotidyl transferase deoxyuridinetriphosphate nick end labelling (TUNEL). Tumor angiogenesis was assessed by IF staining for CD31 and $\alpha$-smooth muscle actin (SMA) [31]. Microscopic images were captured using an UltraVIEW Vox confocal microscope (PerkinElmer, USA). 10 view fields were randomly selected and the fluorescence intensity was analyzed using image $\mathrm{J}$ software, and then the average value was calculated.

\section{Statistical analysis}

Values were expressed as the mean \pm standard deviation (SD) for at least two independent experiments. The two-tailed Student t-test and oneway ANOVA were used for comparisons of two or more than two groups, respectively. Statistical significance was defined as $\mathrm{P}<0.05$. Statistical analysis was performed using GraphPad Prism 5 (GraphPad Software, Inc.).

\section{Abbreviations}

UPP: ubiquitin-proteasome pathway; MM: multiple myeloma; MCL: mantle cell lymphoma; CT-L: chymotrypsin-like; C-L: caspase-like; ER: endoplasmic reticulum; BM: bone marrow; PDX: patient-derived xenograft model; PBMCs: Peripheral blood mononuclear 
cells; T-L: trypsin-like; cCP: constitutive 20S proteasomes; iCP: $20 \mathrm{~S}$ immunoproteasome; TNF- $\alpha$ : tumor necrosis factor- $\alpha$; PN: peripheral neurotoxicity; SAEs: serious adverse events.

\section{AUTHOR CONTRIBUTIONS}

H.Z., M.L., Y.Z., Y.Y. and J.W. designed the research studies. H.Z., M.L., H.Z., J.H., X.W., W.Z., E.I. and C.Z. performed and analyzed in vivo experiments. H.Z., W.W., H.F., Z.L., E.I. and L.Q. performed the in vitro experiments and analyzed the data and L.C. provided cells, H.Z., Y.Z., Y.Y., M.G., E.I, and M.L. wrote or revised the manuscript. Competing interests: The authors declare that they have no competing interests.

\section{CONFLICTS OF INTEREST}

The authors declare no conflicts of interest.

\section{FUNDING}

This work was supported by the following grants: National Natural Science Foundation of China (2187706 to M. L., 121877061 to Y.Z., 81670200 to Y.Y.); Natural Science Foundation of Jiangsu Province (BK20171448 to M. L.); National and Local Joint Engineering Research Center of Biomedical Functional Materials; The 2016 outstanding youth fund of Jiangsu Province (BK20160048 to Y.Y.); National key research and development program-precision medicine subprogram (2016YFC0905900 to Y.Y.). We thank L.C. for kindly providing myeloma cells. We thank E.I. for commenting on the manuscript.

\section{REFERENCES}

1. Rajkumar SV, Richardson PG, Hideshima T, Anderson KC. Proteasome inhibition as a novel therapeutic target in human cancer. J Clin Oncol. 2005; 23:630-39. https://doi.org/10.1200/JCO.2005.11.030 PMID:15659509

2. Orlowski RZ, Kuhn DJ. Proteasome inhibitors in cancer therapy: lessons from the first decade. Clin Cancer Res. 2008; 14:1649-57.

https://doi.org/10.1158/1078-0432.CCR-07-2218 PMID: 18347166

3. Kupperman E, Lee EC, Cao Y, Bannerman B, Fitzgerald M, Berger A, Yu J, Yang Y, Hales P, Bruzzese F, Liu J, Blank J, Garcia K, et al. Evaluation of the proteasome inhibitor MLN9708 in preclinical models of human cancer. Cancer Res. 2010; 70:1970-80. https://doi.org/10.1158/0008-5472.CAN-09-2766 PMID:20160034
4. Roy SS, Kirma NB, Santhamma B, Tekmal RR, Agyin JK. Effects of a novel proteasome inhibitor BU-32 on multiple myeloma cells. Cancer Chemother Pharmacol. 2014; 73:1263-71. https://doi.org/10.1007/s00280-014-2463-3 PMID:24728817

5. Frankland-Searby S, Bhaumik SR. The 26S proteasome complex: an attractive target for cancer therapy. Biochim Biophys Acta. 2012; 1825:64-76. https://doi.org/10.1016/i.bbcan.2011.10.003 PMID:22037302

6. Piva R, Ruggeri B, Williams M, Costa G, Tamagno I, Ferrero D, Giai V, Coscia M, Peola S, Massaia M, Pezzoni G, Allievi C, Pescalli N, et al. CEP-18770: a novel, orally active proteasome inhibitor with a tumorselective pharmacologic profile competitive with bortezomib. Blood. 2008; 111:2765-75.

https://doi.org/10.1182/blood-2007-07-100651 PMID:18057228

7. Drexler HC, Risau W, Konerding MA. Inhibition of proteasome function induces programmed cell death in proliferating endothelial cells. FASEB J. 2000; 14:65-77.

https://doi.org/10.1096/fasebj.14.1.65 PMID:10627281

8. Decker T, Schneller F, Hipp S, Miething C, Jahn T, Duyster J, Peschel C. Cell cycle progression of chronic lymphocytic leukemia cells is controlled by cyclin D2, cyclin D3, cyclin-dependent kinase (cdk) 4 and the cdk inhibitor p27. Leukemia. 2002; 16:327-34. https://doi.org/10.1038/sj.leu.2402389 PMID: 11896535

9. Obeng EA, Carlson LM, Gutman DM, Harrington WJ Jr, Lee KP, Boise LH. Proteasome inhibitors induce a terminal unfolded protein response in multiple myeloma cells. Blood. 2006; 107:4907-16.

https://doi.org/10.1182/blood-2005-08-3531 PMID:16507771

10. Manasanch EE, Orlowski RZ. Proteasome inhibitors in cancer therapy. Nat Rev Clin Oncol. 2017; 14:417-33. https://doi.org/10.1038/nrclinonc.2016.206 PMID:28117417

11. Robak T, Huang H, Jin J, Zhu J, Liu T, Samoilova O, Pylypenko H, Verhoef G, Siritanaratkul N, Osmanov E, Alexeeva J, Pereira J, Drach J, et al, and LYM-3002 Investigators. Bortezomib-based therapy for newly diagnosed mantle-cell lymphoma. N Engl J Med. 2015; 372:944-53.

https://doi.org/10.1056/NEJMoa1412096 PMID:25738670

12. Hideshima T, Richardson P, Chauhan D, Palombella VJ, Elliott PJ, Adams J, Anderson KC. The proteasome inhibitor PS-341 inhibits growth, induces apoptosis, 
and overcomes drug resistance in human multiple myeloma cells. Cancer Res. 2001; 61:3071-76.

PMID:11306489

13. Chen D, Frezza M, Schmitt S, Kanwar J, Dou QP. Bortezomib as the first proteasome inhibitor anticancer drug: current status and future perspectives. Curr Cancer Drug Targets. 2011; 11:239-53.

https://doi.org/10.2174/156800911794519752

PMID:21247388

14. Fuchs O. Targeting of NF-kappaB signaling pathway, other signaling pathways and epigenetics in therapy of multiple myeloma. Cardiovasc Hematol Disord Drug Targets. 2013; 13:16-34.

https://doi.org/10.2174/1871529×11313010003 PMID:23534949

15. Hideshima T, Chauhan D, Richardson P, Mitsiades C, Mitsiades N, Hayashi T, Munshi N, Dang L, Castro A, Palombella V, Adams J, Anderson KC. NF-kappa B as a therapeutic target in multiple myeloma. J Biol Chem. 2002; 277:16639-47.

https://doi.org/10.1074/ibc.M200360200

PMID:11872748

16. Chauhan D, Anderson KC. Mechanisms of cell death and survival in multiple myeloma (MM): therapeutic implications. Apoptosis. 2003; 8:337-43.

https://doi.org/10.1023/a:1024164700094

PMID:12815276

17. Chauhan D, Li G, Shringarpure R, Podar K, Ohtake Y, Hideshima T, Anderson KC. Blockade of Hsp27 overcomes bortezomib/proteasome inhibitor PS-341 resistance in lymphoma cells. Cancer Res. 2003; 63:6174-77.

PMID: 14559800

18. Gu H, Chen X, Gao G, Dong H. Caspase-2 functions upstream of mitochondria in endoplasmic reticulum stress-induced apoptosis by bortezomib in human myeloma cells. Mol Cancer Ther. 2008; 7:2298-307. https://doi.org/10.1158/1535-7163.MCT-08-0186 PMID:18723477

19. Chauhan D, Li G, Hideshima T, Podar K, Mitsiades C, Mitsiades N, Munshi N, Kharbanda S, Anderson KC. JNK-dependent release of mitochondrial protein, smac, during apoptosis in multiple myeloma (MM) cells. J Biol Chem. 2003; 278:17593-96.

https://doi.org/10.1074/jbc.C300076200 PMID:12665525

20. Hao M, Zhang L, An G, Meng H, Han Y, Xie Z, Xu Y, Li C, Yu Z, Chang H, Qiu L. Bone marrow stromal cells protect myeloma cells from bortezomib induced apoptosis by suppressing microRNA-15a expression. Leuk Lymphoma. 2011; 52:1787-94. https://doi.org/10.3109/10428194.2011.576791 PMID:21534877

21. Su Y, Amiri KI, Horton LW, Yu Y, Ayers GD, Koehler E, Kelley MC, Puzanov I, Richmond A, Sosman JA. A phase I trial of bortezomib with temozolomide in patients with advanced melanoma: toxicities, antitumor effects, and modulation of therapeutic targets. Clin Cancer Res. 2010; 16:348-57. https://doi.org/10.1158/1078-0432.CCR-09-2087 PMID:20028756

22. Zang M, Li Z, Liu L, Li F, Li X, Dai Y, Li W, Kuckelkorn U, Doeppner TR, Hermann DM, Zhou W, Qiu L, Jin F. Antitumor activity of the proteasome inhibitor BSc2118 against human multiple myeloma. Cancer Lett. 2015; 366:173-81.

https://doi.org/10.1016/j.canlet.2015.06.011 PMID:26116344

23. Kubiczkova L, Pour L, Sedlarikova L, Hajek R, Sevcikova S. Proteasome inhibitors - molecular basis and current perspectives in multiple myeloma. J Cell Mol Med. 2014; 18:947-61.

https://doi.org/10.1111/jcmm.12279 PMID:24712303

24. Abramson HN. The Multiple Myeloma Drug Pipeline2018: A Review of Small Molecules and Their Therapeutic Targets. Clin Lymphoma Myeloma Leuk. 2018; 18:611-627. https://doi.org/10.1016/j.clml.2018.06.015 PMID:30001985

25. Zhu Y, Zhao X, Zhu X, Wu G, Li Y, Ma Y, Yuan Y, Yang J, $\mathrm{Hu}$ Y, Ai L, Gao Q. Design, synthesis, biological evaluation, and structure-activity relationship (SAR) discussion of dipeptidyl boronate proteasome inhibitors, part I: comprehensive understanding of the SAR of alpha-amino acid boronates. J Med Chem. 2009; 52:4192-99. https://doi.org/10.1021/im9005093 PMID:19537716

26. Zhu Y, Zhu X, Wu G, Ma Y, Li Y, Zhao X, Yuan Y, Yang J, Yu S, Shao F, Li R, Ke Y, Lu A, et al. Synthesis, in vitro and in vivo biological evaluation, docking studies, and structure-activity relationship (SAR) discussion of dipeptidyl boronic acid proteasome inhibitors composed of beta-amino acids. J Med Chem. 2010; 53:1990-99.

https://doi.org/10.1021/jm901407s PMID:20158184

27. Lei M, Feng H, Bai E, Zhou H, Wang J, Shi J, Wang X, Hu $\mathrm{S}$, Liu Z, Zhu Y. Design, synthesis, in vitro and in vivo evaluation, and structure-activity relationship (SAR) discussion of novel dipeptidyl boronic acid proteasome inhibitors as orally available anti-cancer agents for the treatment of multiple myeloma and mechanism studies. Bioorg Med Chem. 2018; 26:3975-81. 
https://doi.org/10.1016/i.bmc.2018.06.020 PMID:29934218

28. Lei $M$, Feng $H$, Bai $E$, Zhou $H$, Wang J, Qin $Y$, Zhang $H$, Wang X, Liu Z, Hai O, Liu J, Zhu Y. Discovery of a novel dipeptidyl boronic acid proteasome inhibitor for the treatment of multiple myeloma and triple-negative breast cancer. Org Biomol Chem. 2019; 17:683-91. https://doi.org/10.1039/c8ob02668h PMID:30601533

29. Arastu-Kapur S, Anderl JL, Kraus M, Parlati F, Shenk KD, Lee SJ, Muchamuel T, Bennett MK, Driessen C, Ball AJ, Kirk CJ. Nonproteasomal targets of the proteasome inhibitors bortezomib and carfilzomib: a link to clinical adverse events. Clin Cancer Res. 2011; 17:2734-43. https://doi.org/10.1158/1078-0432.CCR-10-1950 PMID:21364033

30. Demo SD, Kirk CJ, Aujay MA, Buchholz TJ, Dajee M, Ho MN, Jiang J, Laidig GJ, Lewis ER, Parlati F, Shenk KD, Smyth MS, Sun CM, et al. Antitumor activity of PR-171, a novel irreversible inhibitor of the proteasome. Cancer Res. 2007; 67:6383-91. https://doi.org/10.1158/0008-5472.CAN-06-4086 PMID:17616698

31. Chauhan D, Tian Z, Zhou B, Kuhn D, Orlowski R, Raje N, Richardson $\mathrm{P}$, Anderson $\mathrm{KC}$. In vitro and in vivo selective antitumor activity of a novel orally bioavailable proteasome inhibitor MLN9708 against multiple myeloma cells. Clin Cancer Res. 2011; 17:5311-21.

https://doi.org/10.1158/1078-0432.CCR-11-0476 PMID:21724551

32. Hideshima $T$, Ikeda $H$, Chauhan $D$, Okawa $Y$, Raje $N$, Podar K, Mitsiades C, Munshi NC, Richardson PG, Carrasco RD, Anderson KC. Bortezomib induces canonical nuclear factor-kappaB activation in multiple myeloma cells. Blood. 2009; 114:1046-52. https://doi.org/10.1182/blood-2009-01-199604 PMID:19436050

33. Chauhan $D$, Singh AV, Aujay $M$, Kirk CJ, Bandi $M$, Ciccarelli B, Raje N, Richardson P, Anderson KC. A novel orally active proteasome inhibitor ONX 0912 triggers in vitro and in vivo cytotoxicity in multiple myeloma. Blood. 2010; 116:4906-15.

https://doi.org/10.1182/blood-2010-04-276626 PMID:20805366

34. Pennisi A, Li X, Ling W, Khan S, Zangari M, Yaccoby S. The proteasome inhibitor, bortezomib suppresses primary myeloma and stimulates bone formation in myelomatous and nonmyelomatous bones in vivo. Am J Hematol. 2009; 84:6-14. https://doi.org/10.1002/ajh.21310 PMID:18980173

35. Park J, Park E, Jung CK, Kang SW, Kim BG, Jung Y, Kim $\mathrm{TH}$, Lim JY, Lee SE, Min CK, Won KA. Oral proteasome inhibitor with strong preclinical efficacy in myeloma models. BMC Cancer. 2016; 16:247.

https://doi.org/10.1186/s12885-016-2285-2

PMID:27012957

36. Ettari R, Previti S, Bitto A, Grasso S, Zappalà M. Immunoproteasome-Selective Inhibitors: A Promising Strategy to Treat Hematologic Malignancies, Autoimmune and Inflammatory Diseases. Curr Med Chem. 2016; 23:1217-38.

https://doi.org/10.2174/0929867323666160318173706 PMID:26965184

37. Parlati F, Lee SJ, Aujay M, Suzuki E, Levitsky K, Lorens $J B$, Micklem DR, Ruurs P, Sylvain C, Lu Y, Shenk KD, Bennett MK. Carfilzomib can induce tumor cell death through selective inhibition of the chymotrypsin-like activity of the proteasome. Blood. 2009; 114:3439-47. https://doi.org/10.1182/blood-2009-05-223677 PMID:19671918

38. Schwaid AG, Cornella-Taracido I. Causes and significance of increased compound potency in cellular or physiological contexts. J Med Chem. 2018; 61:1767-73.

https://doi.org/10.1021/acs.jmedchem.7b00762 PMID:28820267

39. Jia D, Koonce NA, Halakatti R, Li X, Yaccoby S, Swain FL, Suva $L$, Hennings L, Berridge MS, Apana SM, Mayo K, Corry PM, Griffin RJ. Repression of multiple myeloma growth and preservation of bone with combined radiotherapy and anti-angiogenic agent. Radiat Res. 2010; 173:809-17.

https://doi.org/10.1667/RR1734.1 PMID:20518660

40. Garcia-Gomez A, Quwaider D, Canavese M, Ocio EM, Tian Z, Blanco JF, Berger AJ, Ortiz-de-Solorzano C, Hernández-Iglesias T, Martens AC, Groen RW, MateoUrdiales J, Fraile $S$, et al. Preclinical activity of the oral proteasome inhibitor MLN9708 in myeloma bone disease. Clin Cancer Res. 2014; 20:1542-54. https://doi.org/10.1158/1078-0432.CCR-13-1657 PMID:24486586

41. Chauhan D, Catley L, Li G, Podar K, Hideshima T, Velankar M, Mitsiades C, Mitsiades N, Yasui H, Letai A, Ovaa $\mathrm{H}$, Berkers C, Nicholson B, et al. A novel orally active proteasome inhibitor induces apoptosis in multiple myeloma cells with mechanisms distinct from bortezomib. Cancer Cell. 2005; 8:407-19.

https://doi.org/10.1016/j.ccr.2005.10.013 PMID:16286248

42. McConkey DJ. Bortezomib paradigm shift in myeloma. Blood. 2009; 114:931-32. https://doi.org/10.1182/blood-2009-06-223230 PMID:19643992 
43. Williamson MJ, Blank JL, Bruzzese FJ, Cao Y, Daniels JS, Dick LR, Labutti J, Mazzola AM, Patil AD, Reimer CL, Solomon MS, Stirling M, Tian Y, et al. Comparison of biochemical and biological effects of ML858 (salinosporamide A) and bortezomib. Mol Cancer Ther. 2006; 5:3052-61.

https://doi.org/10.1158/1535-7163.MCT-06-0185

PMID:17172407

44. Fan H, Angelo NG, Warren JD, Nathan CF, Lin G. Oxathiazolones selectively inhibit the human immunoproteasome over the constitutive proteasome. ACS Med Chem Lett. 2014; 5:405-10. https://doi.org/10.1021/ml400531d PMID:24900849

45. Singh AV, Palladino MA, Lloyd GK, Potts BC, Chauhan $D$, Anderson KC. Pharmacodynamic and efficacy studies of the novel proteasome inhibitor NPI-0052 (marizomib) in a human plasmacytoma xenograft murine model. Br J Haematol. 2010; 149:550-59. https://doi.org/10.1111/i.1365-2141.2010.08144.x PMID:20331453

46. Baumann $\mathrm{P}$, Müller $\mathrm{K}$, Mandl-Weber $\mathrm{S}$, Leban J, Doblhofer R, Ammendola A, Baumgartner R, Oduncu F, Schmidmaier R. The peptide-semicarbazone S-2209, a representative of a new class of proteasome inhibitors, induces apoptosis and cell growth arrest in multiple myeloma cells. Br J Haematol. 2009; 144:875-86.

https://doi.org/10.1111/j.1365-2141.2008.07570.x

PMID:19170678

47. Xu Y, Zhang Z, Li J, Tong J, Cao B, Taylor P, Tang X, Wu $D$, Moran MF, Zeng $Y$, Mao X. The ubiquitinconjugating enzyme UBE2O modulates c-maf stability and induces myeloma cell apoptosis. J Hematol Oncol. 2017; 10:132.

https://doi.org/10.1186/s13045-017-0499-7

PMID:28673317

48. Zhou L, Jiang H, Du J, Li L, Li R, Lu J, Fu W, Hou J. USP15 inhibits multiple myeloma cell apoptosis through activating a feedback loop with the transcription factor NF-kBp65. Exp Mol Med. 2018; 50:1-12. https://doi.org/10.1038/s12276-018-0180-4 PMID:30459344

49. Yata K, Yaccoby S. The SCID-rab model: a novel in vivo system for primary human myeloma demonstrating growth of CD138-expressing Malignant cells. Leukemia. 2004; 18:1891-97. https://doi.org/10.1038/sj.leu.2403513 PMID:15385929 


\section{SUPPLEMENTARY MATERIALS}

\section{Supplementary Figures}
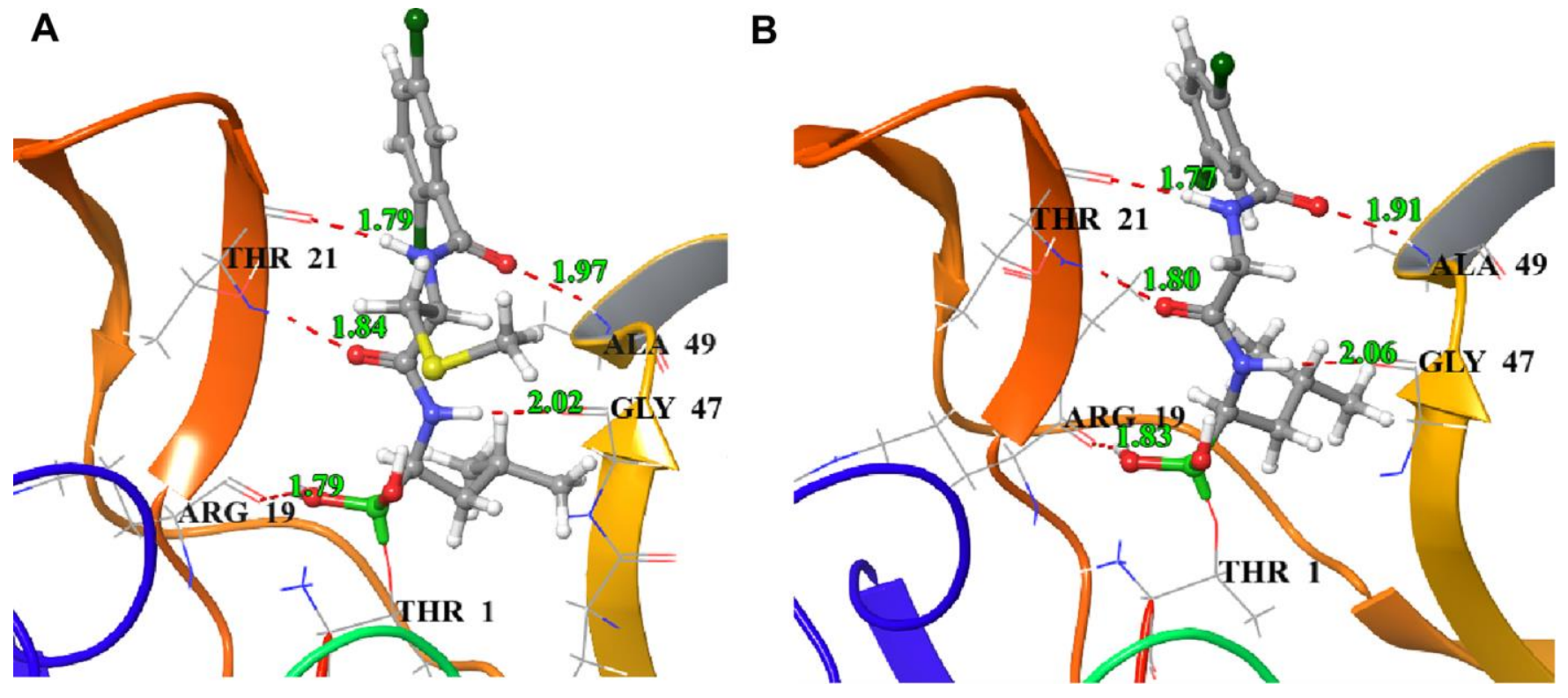

Supplementary Figure 1. Covalent docking with $\beta 5$ subunit. (A) Binding mode of NNU219 with $\beta 5$ subunit; (B) Binding mode of MLN2238 with $\beta 5$ subunit. All the hydrogen atoms were not shown. The amino acid residues of $\beta 5$ subunit were shown in ribbon models. NNU219 and MLN2238 were represented as ball and stick models. The pictures were rendered in PyMOL.
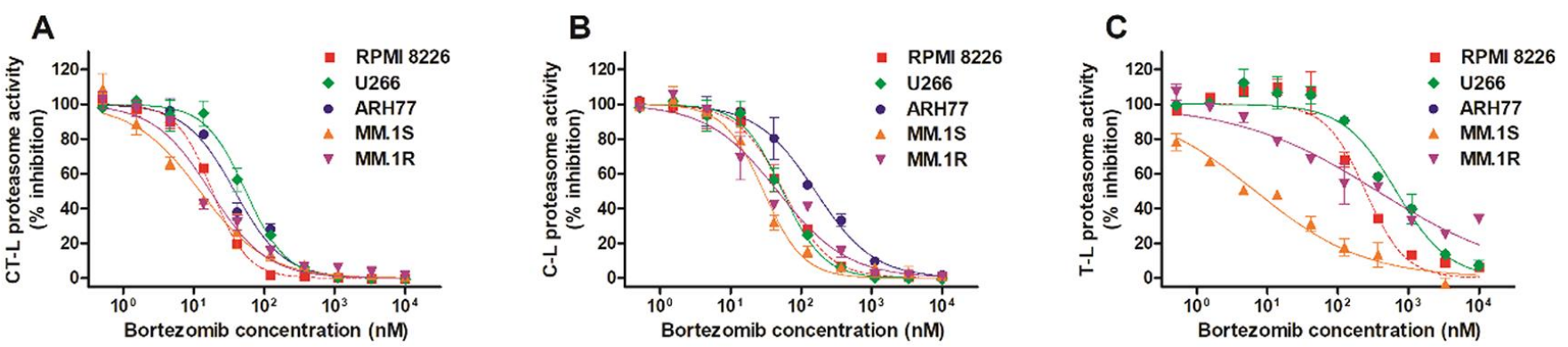

Supplementary Figure 2. Proteasome active site selectivity of bortezomib in human MM cell lines. MM cells were treated with various concentrations of bortezomib for $1 \mathrm{~h}$ and harvested and cytosolic extracts were then analyzed for CT-L (A), C-L (B) and T-L (C) proteasome activity. Results were represented as percent activity of proteasome in drug-treated cells vs. vehicle-treated cells ( \pm SD). 


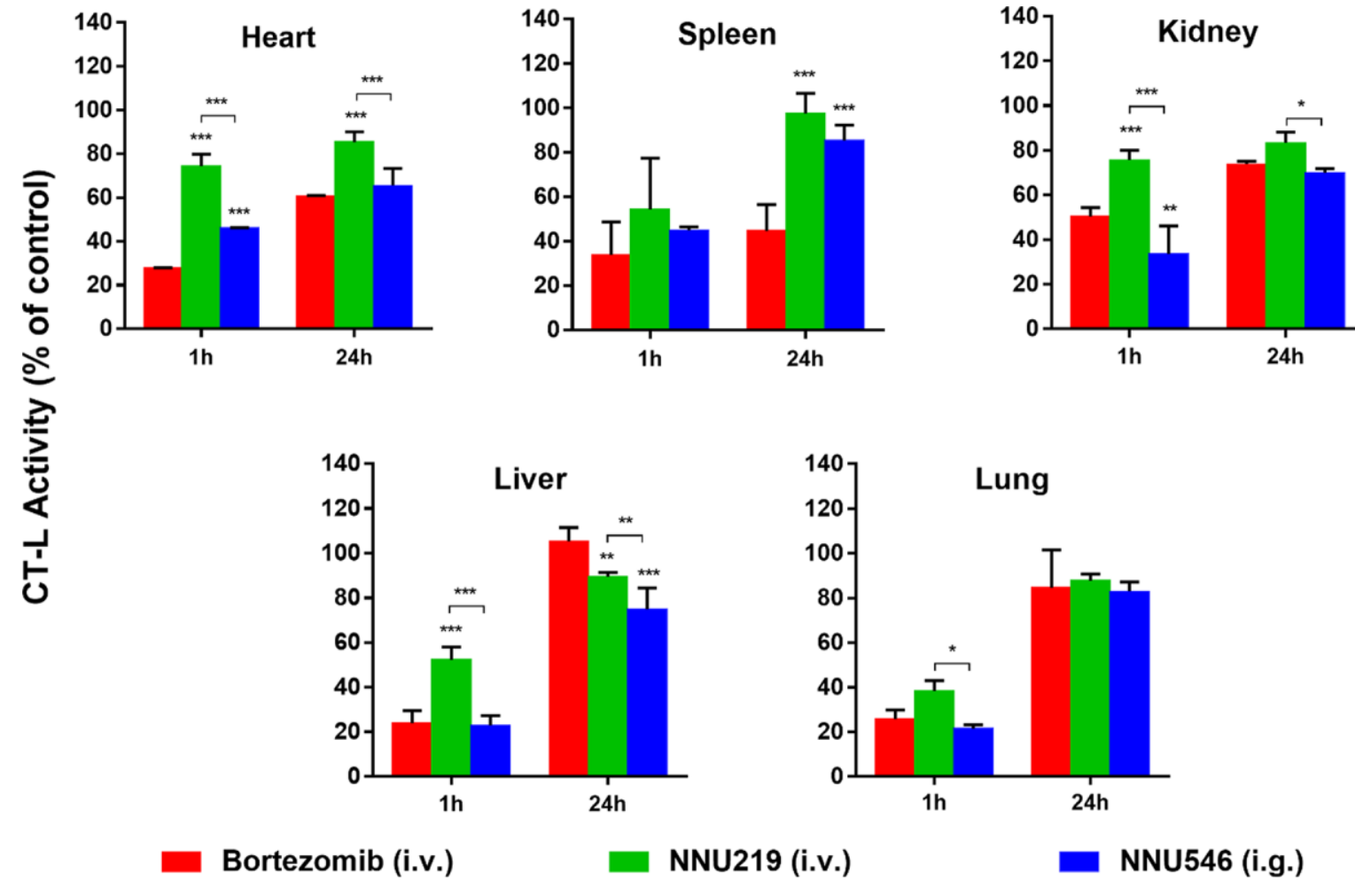

Supplementary Figure 3. Pharmacodynamic profiles of proteasome inhibition in mice bearing human multiple myeloma xenografts. ARH77 tumor-bearing mice were administered with a single dose of NNU219 $(0.4 \mathrm{mg} / \mathrm{kg}, \mathrm{i} . \mathrm{v}$.$) , NNU546 (2 mg/kg, i.g.) or$ bortezomib ( $1 \mathrm{mg} / \mathrm{kg}$, i.v.) and were euthanized at $1 \mathrm{~h}$ and $24 \mathrm{~h}$ after administrations. Blood and normal tissues (spleen, kidney, liver, lung) were harvested and then protein extracts were prepared and the proteasome catalytic activity was evaluated with CT-L subunit-specific fluorescent peptide substrates. Data were presented as the mean \pm SD percent activity relative to vehicle controls ( 3 mice per time-point). $p$ values presented for bortezomib vs NNU219 or NNU546 ( $\left.{ }^{*}, p<0.5 ;{ }^{* *}, p<0.01 ; * * *, p<0.001\right)$. 
A Bone mineral density

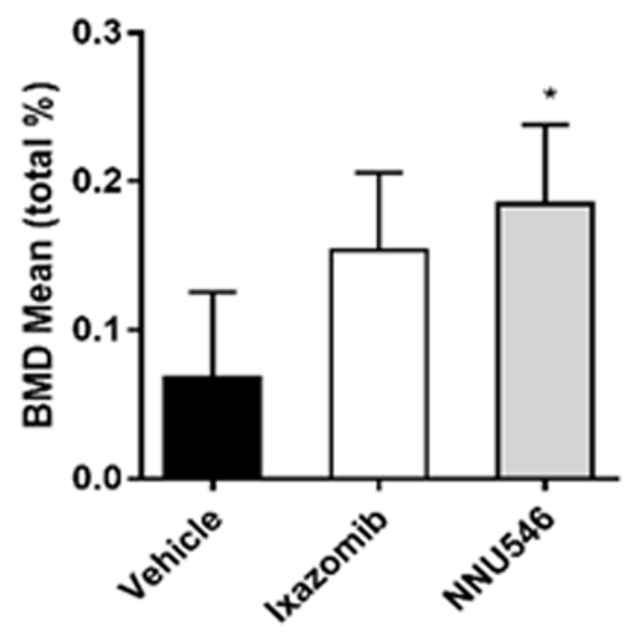

C Trabecular thickness

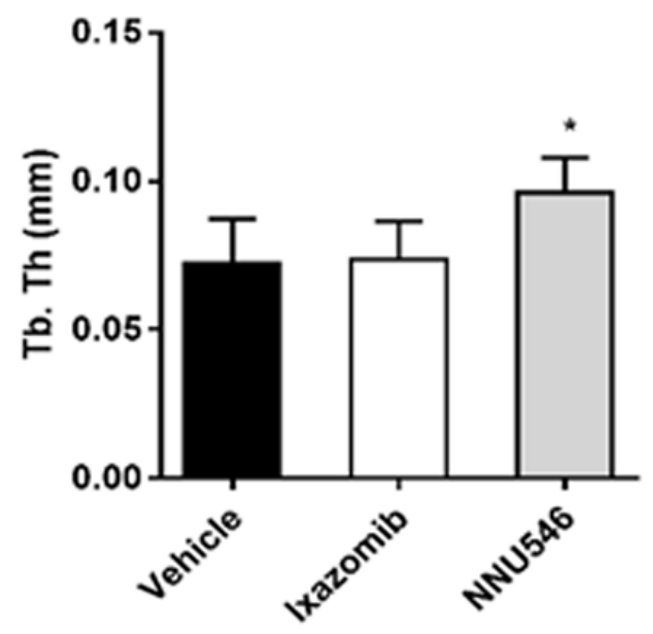

B Trabecular bone volume

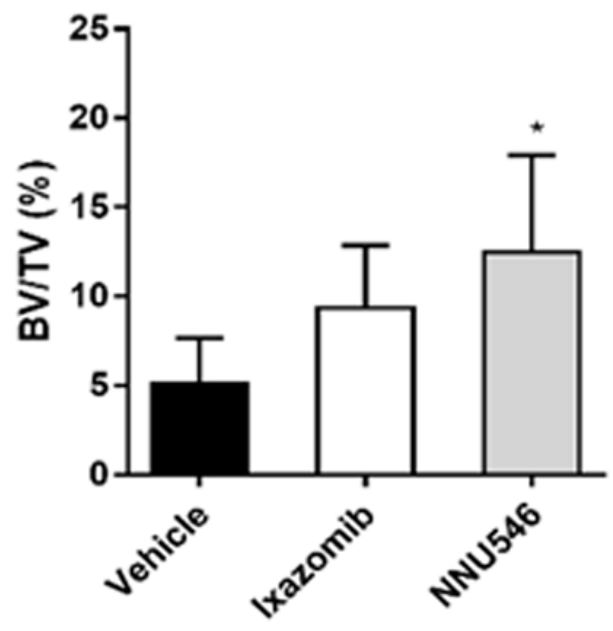

D Trabecular number

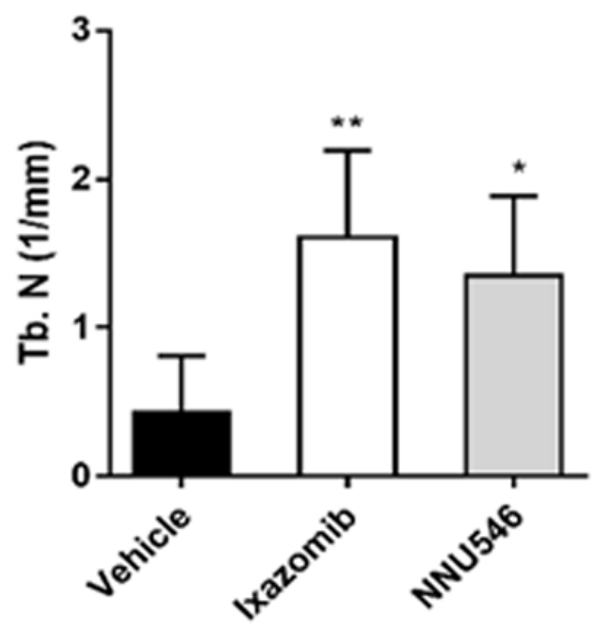

Supplementary Figure 4. NNU546 promoted bone formation in myelomatous bones. SCID-rab mice engrafted with myeloma cells of $\mathrm{MM}$ patients were treated with vehicle, ixazomib $(5 \mathrm{mg} / \mathrm{kg}, \mathrm{BIW})$ or NNU546 (1 mg/kg, QD). Levels of BMD (A) and static histomorphometry (B-D) of the implanted bones at the end of experiment. Data were presented as mean $\pm \mathrm{SD}\left(\mathrm{n}=5 ;{ }^{*}, p<0.05 ; *^{*}, p<0.01\right)$. Y-axis abbreviation: BMD, bone mineral density; BV, bone volume; TV, tissue volume; Tb, trabecular; Th, thickness; N, number. 


\section{Supplementary Tables}

Supplementary Table 1. The microsomal stabilities of the compounds.

\begin{tabular}{|c|c|c|c|c|c|c|}
\hline \multirow{3}{*}{ Compound } & \multicolumn{2}{|c|}{ Human liver microsomes } & \multicolumn{2}{|c|}{ Rat liver microsomes } & \multicolumn{2}{|c|}{ Mouse liver microsomes } \\
\hline & \multirow{2}{*}{$\mathbf{t}_{1 / 2}(\min )$} & \multirow{2}{*}{$\begin{array}{c}\text { CLint } \\
(\mathrm{mL} / \mathrm{min} / \mathrm{kg})\end{array}$} & \multirow{2}{*}{$\mathbf{t}_{1 / 2}(\min )$} & \multirow{2}{*}{$\begin{array}{c}\text { CLint } \\
(\mathrm{mL} / \mathrm{min} / \mathrm{kg})\end{array}$} & \multirow{2}{*}{$\mathbf{t}_{1 / 2}(\min )$} & \multirow{2}{*}{$\begin{array}{c}\text { CLint } \\
(\mathrm{mL} / \mathrm{min} / \mathrm{kg})\end{array}$} \\
\hline & & & & & & \\
\hline Bortezomib & 21.4 & 58.3 & 12.4 & 200.5 & 10.7 & 508.7 \\
\hline NNU219 & 32.4 & 43 & 27.8 & 50 & 16.7 & 83 \\
\hline
\end{tabular}

Note: The microsomal stabilities of bortezomib and NNU219 were determined in the presence of pooled human, rat or mouse liver microsomes. $\mathrm{T}_{1 / 2}(\mathrm{~min})$ and $\mathrm{CL}_{\text {int }}(\mathrm{mL} / \mathrm{min} / \mathrm{kg})$ were detected by $\mathrm{LC} / \mathrm{MS}$. Antipyrine and testosterone $(5 \mu \mathrm{M}$ each) were used as positive and negative controls, respectively. Abbreviations: $t=$ time; $\mathrm{CL}_{\text {int, }}$ intrinsic clearance.

Supplementary Table 2. Pharmacokinetic profiles of NNU546 in mice.

\begin{tabular}{lcccccc}
\hline & $\mathbf{t}_{\mathbf{1} / \mathbf{2}}(\mathbf{h})$ & $\mathbf{C L}\left(\mathbf{m L} \cdot \mathbf{h}^{-\mathbf{1}} \cdot \mathbf{k g}^{-\mathbf{1}}\right)$ & $\mathbf{V z}\left(\mathbf{m L} \cdot \mathbf{k g}^{-\mathbf{1}}\right)$ & $\mathbf{A U C} \mathbf{0 - t}\left(\mathbf{h} \cdot \mathbf{n g} \cdot \mathbf{m L}^{-\mathbf{1}}\right)$ & MRT $(\mathbf{h})$ & F $(\boldsymbol{\%})$ \\
\hline i.v. & $2.08 \pm 0.991$ & $986 \pm 75.4$ & $2914 \pm 1247$ & $1890 \pm 73.8$ & $1.55 \pm 0.133$ \\
i.g. & $2.41 \pm 0.420$ & --- & --- & $208 \pm 26.7$ & $2.35 \pm 0.221$ & $11.0 \pm 1.41$ \\
\hline
\end{tabular}

Note: Blood samples were collected at baseline and after intravenous or oral administration of $2 \mathrm{mg} / \mathrm{kg}$ of NNU546. Each time-point represents the average value of three animals. NNU546 concentration in blood and plasma samples was determined by liquid chromatography-tandem mass spectrometry (LC/MS/MS) in a non-good laboratory practice lab. Pharmacokinetic analysis of the blood and plasma concentration data was performed using WinNonlin version 5.2 (Pharsight Corp.). Kinetic parameters were estimated using a noncompartmental model with sparse sampling mode (model 201 for plasma and blood). Area under the concentration vs. time curve (AUC) was calculated using the linear trapezoidal rule. Abbreviations: $t=$ time; $C L$, clearance; $V z$, apparent volume of distribution; $A U C_{0-t}, A U C$ (area under the curve) from 0 to $t h$; $\mathrm{MRT}$, mean residence time; F\%, oral bioavailability. 